\title{
Review \\ Comparative Risk of Bleeding of Anticoagulant Therapy with Vitamin K Antagonists (VKAs) and with Non-Vitamin K Antagonists in Patients Undergoing Dental Surgery
}

\author{
Mattia Manfredini + (D), Pier Paolo Poli ${ }^{+}(\mathbb{D}$, Luca Creminelli, Alberto Porro *, Carlo Maiorana and Mario Beretta \\ Fondazione IRCCS Ca' Granda Ospedale Maggiore Policlinico, Implant Center for Edentulism and Jawbone \\ Atrophies, Maxillofacial Surgery and Odontostomatology Unit, University of Milan, Via della Commenda 10, \\ 20122 Milan, Italy; mattiamanfredinidr@gmail.com (M.M.); PierPaolo.Poli@unimi.it (P.P.P.); \\ luca.creminelli@unimi.it (L.C.); carlo.maiorana@unimi.it (C.M.); Mario.Beretta@unimi.it (M.B.) \\ * Correspondence: Albertoporro1995@gmail.com; Tel.: +39-33-3893-2223 \\ + They should be considered in equal contribution.
}

check for

updates

Citation: Manfredini, M.; Poli, P.P.; Creminelli, L.; Porro, A.; Maiorana, C.; Beretta, M. Comparative Risk of Bleeding of Anticoagulant Therapy with Vitamin K Antagonists (VKAs) and with Non-Vitamin K Antagonists in Patients Undergoing Dental Surgery. J. Clin. Med. 2021, 10, 5526. https://doi.org/10.3390/jcm10235526

Academic Editor: Bruno Chrcanovic

Received: 24 October 2021

Accepted: 22 November 2021

Published: 25 November 2021

Publisher's Note: MDPI stays neutral with regard to jurisdictional claims in published maps and institutional affiliations.

Copyright: (c) 2021 by the authors. Licensee MDPI, Basel, Switzerland. This article is an open access article distributed under the terms and conditions of the Creative Commons Attribution (CC BY) license (https:/ / creativecommons.org/licenses/by/ $4.0 /)$.

\begin{abstract}
Objectives: A wide variety of approaches have been proposed to manage anticoagulant drugs in patients undergoing dental surgery; vitamin $\mathrm{K}$ antagonists and novel direct oral anticoagulants have been used. The present study aims to explore the existing evidence concerning the management of patients in anticoagulant therapy undergoing oral surgery procedures and to give suggestions related to peri- and post-operative measures. Materials and methods: A comprehensive search of databases was conducted to identify studies that evaluated the relationship between direct oral anticoagulants and dental procedures. The present scoping review was realized in adherence with the Preferred Reporting Items for Systematic reviews and Meta-Analyses extension for Scoping Reviews (PRISMA-ScR) guidelines. The publications varied from randomized controlled trials (RCT) to cohort trials. Only articles written in English language and published between 2000 to 2020 were screened. The studies were included if discussing the management of a patient in anticoagulant therapy (warfarin or direct oral anticoagulants) scheduled for tooth extraction. Results: 33 studies were selected and included in the qualitative review. Nineteen considered anticoagulant therapy with warfarin, six considered anticoagulant therapy with new oral anticoagulants and eight compared patients taking warfarin with patients taking direct oral anticoagulants. Conclusions: No case of extractive surgery should alter the posology of the drug: thromboembolic risks derived from discontinuation are heavier than hemorrhagic risks. Clinical relevance: direct oral anticoagulants are safer in terms of bleeding and manageability and bleeding episodes are manageable with local hemostatic measures.
\end{abstract}

Keywords: oral anticoagulants; NOAC; oral surgery; dental extractions; dental surgery; hemostatic measures

\section{Introduction}

As the average age of the population increases, more elderly patients need to be referred for oral surgery, even if it is a simple tooth extraction. These patients often have a medical history and ongoing drug therapy.

The two main types of anticoagulants are Vitamin $\mathrm{K}$ antagonists (VKAs) and the new oral anticoagulants (NOACs), known also as direct oral anticoagulants (DOACs). The most important VKAs are Warfarin and acenocoumarol. There are derivatives of 4-hydroxycoumarin. The targets of VKAs are factors VII, IX, X and proteins C, S and Z [1]. Warfarin therapy reduces the risk of arterial thromboembolic events such as strokes by $70 \%$ [2] and the risk of recurrent venous thromboembolism by $90 \%$ [3]. The anticoagulative effect develops after a latency of $\sim 48 \mathrm{~h}$ and takes 4 to 5 days to be fully active with a half-life of at least $48 \mathrm{~h}$ [4]. This means that the anticoagulant effect persists even 
after discontinuation for a comparatively long time and that must be considered before planning any surgery [5]. To increase and accelerate the anticoagulant effect, the dosage can be raised to a maximum of $75 \mathrm{mg} / \mathrm{kg}$, above which the effect will no longer be accelerated [6]. Prothrombin time (PT) is a blood test that quantifies the time it takes for a fibrin clot to form. PT is usually expressed by an index called INR (International Normalized Ratio). The INR value under normal conditions is $0.9 / 1.2$, but the physician can establish optimal values tailored to the patient. Many guidelines describe the desirable value of the INR for patients taking oral anticoagulants, ranging between 2 and 3. Some studies have demonstrated that exodontias performed in patients with a recommendable INR range can be safely conducted without oral anticoagulation interruption or antiplatelet drugs $[7,8]$. Those recommendations include a reduction, a temporarily interruption of OAT (oral anticoagulant therapy) or a substitution (bridging) with heparin prior to surgical procedure [9-11].

The new oral anticoagulants, instead, act as selective inhibitors of factor II, $\mathrm{X}$ or platelet aggregation inhibitors). Differently from VKAs, DOACs have a more manageable posology and have no need for laboratory tests to monitor INR fluctuations. Selectively blocking the cascade factors of coagulation, these drugs impede the conversion of fibrinogen into fibrin strands, with an effective anticoagulant action [12].

These drugs represent a risk in oral surgery, especially in extractive surgery. The risks range from simple obstruction of the surgeon's field of vision to the possibility of bleeding episodes due to anticoagulation. Bleeding episodes are also favored in extractive surgery because of second intention healing of the socket, and it is therefore necessary to supplement the procedure with effective local hemostatic measures. In patients treated with these molecules and scheduled for oral surgery procedures, scientific evidence for withdrawal, reduction or continuation of the drugs is limited. Kwak et. Al., in a retrospective study, remarked the importance of the type of surgery, finding that the discontinuation of anticoagulant therapy with DOACs (rivaroxaban, dabigatran, apixaban, edoxaban) should change depending on the type of surgery (implant insertion, tooth extractions); nonetheless, the bleeding risk was under control in 144 cases out of 153 [13]. In a recent systematic review, J. Chahine et al. evaluated five RCTs and five case-control studies. The population considered was formed by patients in therapy with VKAs and with NOACs, that required an oral surgery procedure, namely, tooth extraction or implant placement (the two most performed oral surgeries). The main conclusion was that VKAs must be continued in all surgical procedures if INR is in the therapeutic range. As for NOACs, they must also be maintained in most procedures. Local hemostatic agents are mostly needed in both cases [14]. However, out of 10 studies, only one included patients in therapy with NOACs. For such reason, additional evidence is needed to understand how to manage dental patients in therapy with NOACs from a pharmacological standpoint.

In view of the above, the present study aims to explore the existing evidence concerning the management of patients in anticoagulant therapy undergoing oral surgery procedures and to give suggestions related to peri- and post-operative measures. Studies were screened to compare different types of anticoagulant drugs.

\section{Materials and Methods}

\subsection{Protocol}

The present scoping review was realized in adherence with the Preferred Reporting Items for Systematic reviews and Meta-Analyses extension for Scoping Reviews (PRISMAScR) guidelines in order to map evidence on a topic and identify main concepts and knowledge gaps [15]. The protocol of the present scoping review was registered at the National Institute for Health Research PROSPERO, International Prospective Register of Systematic Reviews (https:/ / www.crd.york.ac.uk/PROSPERO, accessed on 15 March 2021 with the registration ID: CRD42021235862.

An adaptation of the PICO (Population, Intervention, Comparison and Outcome) model was used to build a focused question, consisting of a PEO (Population, Exposure, 
and Outcome) framework, to determine the association between a particular exposure and the outcomes [16]. This approach has been developed to perform qualitative systematic reviews in healthcare interventions [17] including oral surgery procedures [18]. The focused question was: "in patients scheduled for tooth extraction, in therapy with anticoagulant drugs including coumarin derivates (warfarin) or new oral anticoagulants (dabigatran, rivaroxaban, edoxaban, apixaban), how frequent are the bleeding events and which is the most adequate posology of the drug in terms of suspension or continuation, in the days approaching the surgery?". The primary outcome was the occurrence of bleeding events, while the secondary outcome was the evaluation of other post-operative complications.

\subsection{Eligibility Criteria}

\subsubsection{Inclusion Criteria}

To be included, every source of evidence had to fulfil specific inclusion criteria. Only articles written in English language and published between 2000 to 2020 were screened. Studies included in the screening process were randomized controlled trials (RCTs), controlled clinical trials (CCTs), retrospective and prospective case-control studies. No restrictions were placed on population characteristics, number of patients, age or systemic conditions. The studies were included if discussing the management of a patient in anticoagulant therapy (warfarin or DOACs) scheduled for tooth extraction (some studies considered other types of surgery), including the pre-operative pharmacological protocol, the post-operative measures, the number and the risk of bleeding events.

\subsubsection{Exclusion Criteria}

All studies not fulfilling the inclusion criteria were excluded, such as (1) articles written in a language other than English; (2) case series, case reports, literature reviews; (3) studies that did not report neither the pharmacological management nor the evaluation of the bleeding risk; and (4) studies that did not add enough relevant data related to the topic.

\subsection{Sources of Evidence}

An electronic literature search was conducted in PubMed via MEDLINE, Scopus and Web of Science databases. The electronic search was complemented by a manual search of the reference lists of all selected full-text articles. The search aimed to collect relevant information about the perioperative measures and the changing in the posology in patients under anticoagulant therapy. In all databases, the years covered were from 2000 to 2020. The most recent research was executed on 10 January 2021.

\subsection{Search Strategies}

For all the libraries, a combination of specific keywords, medical subject headings $[\mathrm{MeSH}]$ and other terms not indexed as MeSH were used to identify all pertinent studies, according to the precise indications of the PEO question. As such, for the PubMed library, articles were selected using the following terms: (Anticoagulants[MeSH Terms]) AND (Surgery, Oral[MeSH Terms]). For the Scopus library, the terms used were dental AND surgery AND dabigatran AND rivaroxaban AND apixaban AND edoxaban AND warfarin. Finally, the terms used in the Web of Science library were TOPIC: (direct oral anticoagulants) AND TOPIC: (dental surgery) AND TOPIC: (warfarin). In each research string, some filters were applied, such as type of publication (randomized controlled study, retrospective study, controlled study) and year of publication (2000-2020).

\subsection{Selection of Sources of Evidence}

Two reviewers (A.P. and L.C.), working independently, completed the initial screening of titles and abstracts of all included papers. Full-text articles were assessed independently, and the selections compared between the two investigators. The final list and every disagreement between the two investigators were brought to the attention of a third and 
fourth investigator (M.M. and P.P.P.). Duplicate articles in the databases were identified and removed using EndNote Web reference manager software (Clarivate Analytics, Philadelphia, PA, USA). Some authors of the studies included were contacted to obtain unavailable full texts or to clarify uncertain and incomplete data.

\subsection{Data Charting Process and Data Items}

During the review process, an electronic spreadsheet (Microsoft Excel ${ }^{\circledR}$, Redmond, WA, USA) was created and consecutively updated. All the useful information in each study was collected in tables. The demographic data were divided in categories: first author name, year of publication, title, type of study, database in which the study was found, number of patients participating, number of teeth extracted. Tables describing the primary and secondary outcome, the method of measurement of the primary outcome (bleeding events), the number and type of bleeding events were completed, along with tables containing all the data about the anticoagulant therapy including type of anticoagulant therapy (warfarin, DOACs, or both), suspension or continuation of the therapy, and other drugs taken by the patients. Additional tables were filled with information about comorbidities, time of post-operative follow-up, the eligibility criteria adopted in each study and the authors' conclusions.

\subsection{Methodological and Reporting Quality Assessment}

Due to the specific research question herein, aiming to summarize the risk and the measure to consider in a patient taking oral anticoagulants and about to undergo a tooth extraction, no study quality assessment was performed. This is in accordance with the PRISMA-ScR guidelines stating that the risk of bias evaluation across studies is not applicable for scoping reviews.

\subsection{Synthesis of Results}

All data collection was done using a spreadsheet designed to express all the data regarding study characteristics and outcomes as tables in the results. As no meta-analysis was carried out due to the qualitative nature of the present scoping review and the impossibility to obtain an objective method of measurement of the primary outcome (bleeding events) evaluated in each study, a descriptive qualitative statistical approach was used to present the data.

\section{Results}

\subsection{Selection of Sources of Evidence}

Initially, 432 articles were found. Overall, 406 articles were identified in PubMed, nine in Scopus and 17 in Web of Science. Once all the duplicates were removed and the records were screened, 51 studies remained available. After applying the inclusion criteria, a total of 37 articles were identified. The final number of manuscripts included in the qualitative synthesis was 33. The flow diagram of the search and selection process is shown in Figure 1 and the studies were divided following the drug typology in Tables 1-3.

\subsection{Characteristics of Sources of Evidence}

Of the 33 studies included, 19 [4,19-39] considered patients in therapy with VKAs (warfarin and acenocoumarol) (Table 1).

A total of six studies [13,40-46] involved patients in therapy with NOACs (Table 2). In this category, four different types of drug were used for anticoagulation therapy: dabigatran, rivaroxaban, apixaban and edoxaban.

The remaining eight studies [47-52] were the most relevant to the aim of the present review, as they compared patients treated with VKAs with patients in therapy with NOACs (Table 3). 


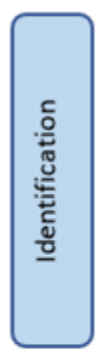

Records identified after database searching $(n=432)$

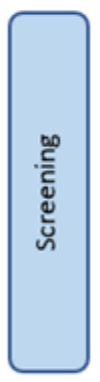

Records after duplicates removed and screened

$(n=51)$

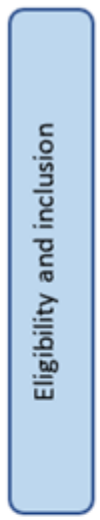

Full texts articles assessed for eligibility $(n=37)$
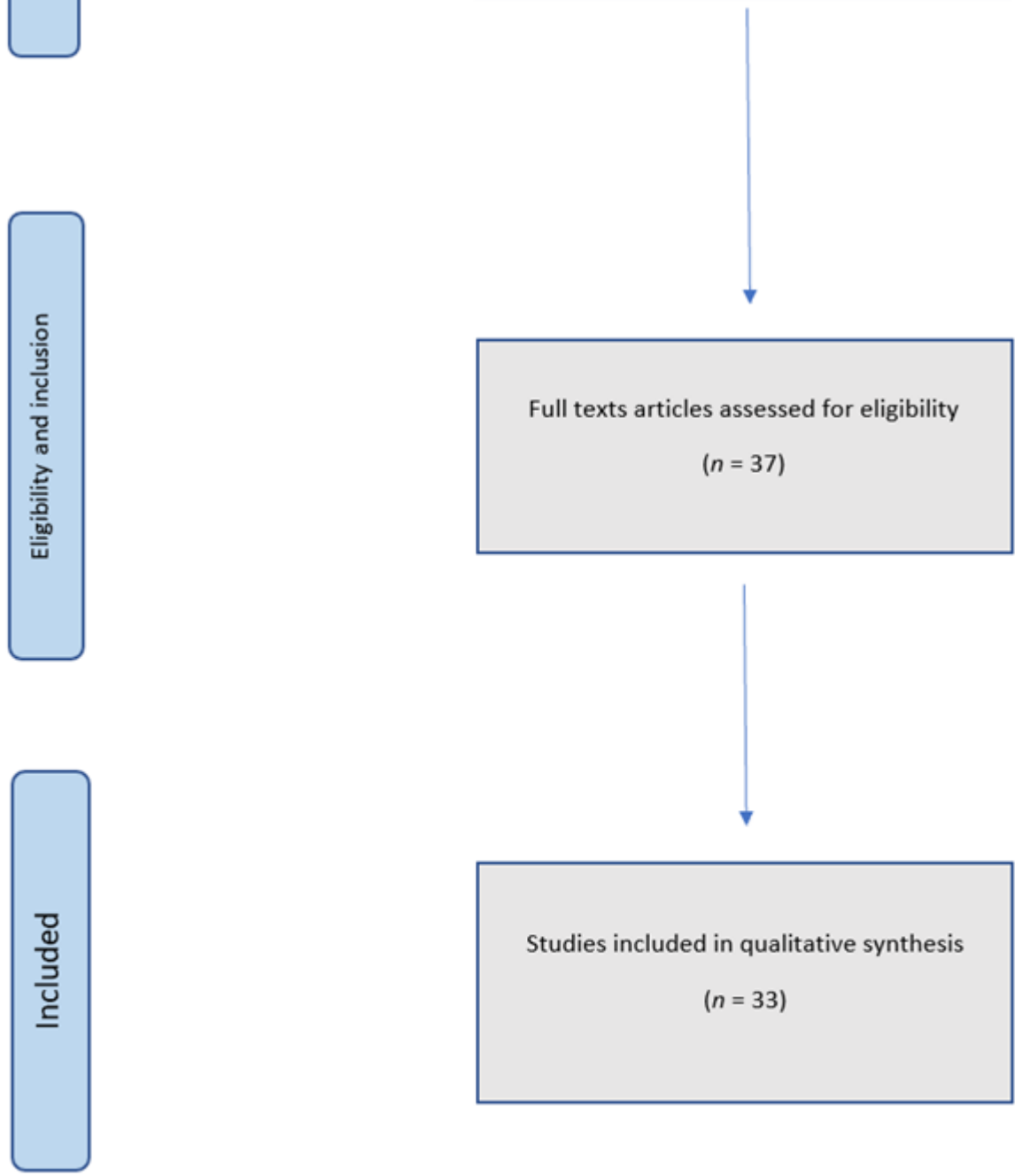

Studies included in qualitative synthesis

$$
(n=33)
$$

Figure 1. Flow chart of inclusion and selection. 
Table 1. Studies considering VKAs.

\begin{tabular}{|c|c|c|c|c|c|c|c|c|c|}
\hline Author & $\begin{array}{l}\text { Description of } \\
\text { Primary Outcome }\end{array}$ & Anticoagulant & $\begin{array}{l}\text { Sospension of } \\
\text { Anticoagulant }\end{array}$ & Other Drugs & $\begin{array}{l}\text { Type of Oral } \\
\text { Surgery } \\
\text { Procedure }\end{array}$ & $\begin{array}{l}\text { Monitoring } \\
\text { Time and } \\
\text { Follow Up }\end{array}$ & $\begin{array}{l}\text { Method of Bleeding } \\
\text { Control }\end{array}$ & $\begin{array}{l}\text { Number of } \\
\text { Partecipants }\end{array}$ & $\begin{array}{l}\text { Number and Type } \\
\text { of Bleeding Events }\end{array}$ \\
\hline $\begin{array}{l}\text { B. Bajkin, I. } \\
\text { Bajkin, B. } \\
\text { Petrovic. }\end{array}$ & $\begin{array}{l}\text { Lockhart definition } \\
\text { of bleeding event: } \\
\text { bleeding wich } \\
\text { continues for more } \\
\text { than } 12 \mathrm{~h} \text {; the patient } \\
\text { returns to dental } \\
\text { practice or } \\
\text { emergency } \\
\text { department; large } \\
\text { hematoma or } \\
\text { ecchymosis within } \\
\text { the oral soft tissues; } \\
\text { need of blood } \\
\text { transfusion. }\end{array}$ & Acenocoumarol. & No. & $\begin{array}{l}\text { Antibiotic; } \\
\text { Painkiller, if } \\
\text { necessary. }\end{array}$ & $\begin{array}{c}\text { Teeth } \\
\text { extraction. }\end{array}$ & $\begin{array}{l}2 \mathrm{~h} \text {; follow up } \\
5 \text { days, with } \\
\text { day } 1 \text { and day } \\
2 \text { controls. }\end{array}$ & $\begin{array}{l}\text { Collagen sponge in } \\
\text { extraction sockets; no } \\
\text { suture; gauze pressure } \\
\text { for } 30^{\prime} \text {. In case of } \\
\text { post-operative } \\
\text { bleeding: gauze } \\
\text { compression and } \\
\text { eventually suture; } \\
\text { administration of } \\
\text { vitamin K, } \\
\text { prothrombin complex } \\
\text { concentrate or } \\
\text { fresh frozen plasma. }\end{array}$ & 213 & $\begin{array}{c}\text { Postoperative } \\
\text { bleeding: } 3 \text { in group } \\
\text { anticoagulant- } \\
\text { aspirin; } 2 \text { in group } \\
\text { only anticoagulant; } \\
\text { no in aspirin group. }\end{array}$ \\
\hline $\begin{array}{l}\text { B. Bajkin, S. } \\
\text { Popovic, S. } \\
\text { Selakovic. }\end{array}$ & $\begin{array}{l}\text { Hemorrhage: event } \\
\text { requiring local } \\
\text { pressure or } \\
\text { additional surgical } \\
\text { intervention. } \\
\text { Immediate (first } 2 \mathrm{~h} \text { ) } \\
\text { or late. }\end{array}$ & $\begin{array}{l}\text { Acenocoumarol; } \\
\text { Warfarin }\end{array}$ & $\begin{array}{l}\text { Yes; Stop 3-4 } \\
\text { days before } \\
\text { (INR of }<1.5 \text { ); } \\
\text { Bridging with } \\
\text { heparin; Stop } \\
\text { heparin } 12 \mathrm{~h} \\
\text { before; } \\
\text { Resumed on } \\
\text { evening. }\end{array}$ & $\begin{array}{l}\text { Antibiotic; } \\
\text { Painkiller, if } \\
\text { necessary. }\end{array}$ & $\begin{array}{c}\text { Teeth } \\
\text { extraction. }\end{array}$ & $\begin{array}{l}2 \mathrm{~h} \text { after } \\
\text { surgery; } \\
\text { follow up } 7 \\
\text { days, with day } \\
\text { 1, day 2, day } 4 \\
\text { controls. }\end{array}$ & $\begin{array}{l}\text { Gauze compression for } \\
30 \text {; ; resorbable } \\
\text { collagen sponges. In } \\
\text { case of post-extraction } \\
\text { bleeding, haemostatic } \\
\text { agents and suture; } \\
\text { vitamin K therapy. }\end{array}$ & 214 & $\begin{array}{l}\text { Immediate bleeding } \\
\text { (gauze } \\
\text { compression): } 8 \text { in } \\
\text { the continuation } \\
\text { group; } 5 \text { in the stop } \\
\text { group. second } \\
\text { manifested } \\
\text { post-extraction } \\
\text { bleeding. No } \\
\text { thromboembolic } \\
\text { complications. }\end{array}$ \\
\hline $\begin{array}{l}\text { R. Sacco, M. } \\
\text { Sacco, M. } \\
\text { Carpenedo, } \\
\text { P. Mannucci. }\end{array}$ & $\begin{array}{l}\text { 1- Mild bleeding: < } \\
\text { 10'; 2-Moderate: } \\
\text { 10-20'; 3-Severe: } \\
\text { requiring a new } \\
\text { surgical intervention } \\
\text { or transfusion. }\end{array}$ & $\begin{array}{l}\text { Acenocoumarol; } \\
\text { Warfarin. }\end{array}$ & $\begin{array}{l}\text { Yes, in control } \\
\text { group: dose } \\
\text { reduction (INR } \\
1.8 \text { mantained } \\
\text { up to } 24 \mathrm{~h} \text { after } \\
\text { surgery); full } \\
\text { anticoagulant } \\
\text { dosage resumed } \\
48 \text { h later. No, in } \\
\text { test group } \\
(\text { INR }>2.5) .\end{array}$ & $\begin{array}{l}\text { Antibiotic; } \\
\text { Painkiller, if } \\
\text { necessary. }\end{array}$ & $\begin{array}{c}\text { Teeth } \\
\text { extractions } \\
\text { (nontraumatic } \\
\text { technique, flap } \\
\text { elevation if } \\
\text { necessary); } \\
\text { Removal of } \\
\text { cystic } \\
\text { formations; } \\
\text { Insertion of } \\
\text { implants. }\end{array}$ & $\begin{array}{c}120^{\prime} \text { after } \\
\text { surgery; } \\
\text { Follow up } 6 \\
\text { days } \\
\text { (contacted } \\
\text { daily by } \\
\text { phone); day 7, } \\
\text { suture } \\
\text { removal. }\end{array}$ & $\begin{array}{l}\text { Control group: no } \\
\text { local or general } \\
\text { hemostatic measures; } \\
\text { Test group: gelatin and } \\
\text { oxidized cellulose } \\
\text { sponges; tranexamic } \\
\text { acid (local applications } \\
\text { and mouthwashes, } \\
\text { 4/day for } 2 \text { days). In } \\
\text { case of bleeding: new } \\
\text { suture; removal of } \\
\text { clots; tranexamic acid } \\
\text { mouthwashes. }\end{array}$ & 131 & $\begin{array}{l}\text { No bleeding in the } \\
\text { first } 24 \text { h; Mild } \\
\text { postsurgical: } 10 \\
\text { cases }(15.1 \%) \text { in } \\
\text { control group and } \\
\text { in } 6 \text { cases }(9.2 \%) \text { in } \\
\text { the test group (9 } \\
\text { warfarin and } 7 \\
\text { acenocoumarol); No } \\
\text { severe bleeding. }\end{array}$ \\
\hline
\end{tabular}


Table 1. Cont.

\begin{tabular}{|c|c|c|c|c|c|c|c|c|c|}
\hline Author & $\begin{array}{l}\text { Description of } \\
\text { Primary Outcome }\end{array}$ & Anticoagulant & $\begin{array}{l}\text { Sospension of } \\
\text { Anticoagulant }\end{array}$ & Other Drugs & $\begin{array}{c}\text { Type of Oral } \\
\text { Surgery } \\
\text { Procedure }\end{array}$ & $\begin{array}{c}\text { Monitoring } \\
\text { Time and } \\
\text { Follow Up } \\
\end{array}$ & $\begin{array}{l}\text { Method of Bleeding } \\
\text { Control }\end{array}$ & $\begin{array}{l}\text { Number of } \\
\text { Partecipants }\end{array}$ & $\begin{array}{l}\text { Number and Type } \\
\text { of Bleeding Events }\end{array}$ \\
\hline $\begin{array}{l}\text { P. Cannon, V. } \\
\text { Dharmar. }\end{array}$ & & $\begin{array}{c}\text { Warfarin } \\
\text { (1.5-7.5 mg, } \\
\text { mean } 3.9 \mathrm{mg}) \text {. }\end{array}$ & $\begin{array}{l}\text { Yes, in control } \\
\text { group; Resumed } \\
\text { on the same day. }\end{array}$ & $\begin{array}{c}\text { Antibiotic } \\
\text { prophylaxis if } \\
\text { necessary; } \\
\text { Paracetamol if } \\
\text { necessary; } \\
\text { Avoid aspirin } \\
\text { and } \\
\text { nonsteroidal } \\
\text { antiinflamma- } \\
\text { tory } \\
\text { drugs. }\end{array}$ & $\begin{array}{c}\text { Teeth } \\
\text { extraction } \\
\text { (sometimes } \\
\text { with flap } \\
\text { elevation). }\end{array}$ & $\begin{array}{c}30^{\prime} \text { after } \\
\text { surgery; } \\
\text { follow up } 7 \\
\text { days with day } \\
3 \text { and day } 5 \\
\text { controls. }\end{array}$ & $\begin{array}{l}\text { Control group: local } \\
\text { haemostatic agent } \\
\text { Surgicel }{ }^{\circledR} \text { and } 3 / 0 \\
\text { catgut suture (in study } \\
\text { group only if } \\
\text { involving bone or soft } \\
\text { tissue). Gauze } \\
\text { compression for } 20^{\prime} \text {. }\end{array}$ & 70 & $\begin{array}{c}0 \text { immediate } \\
\text { post-operative } \\
\text { bleeding during the } \\
30^{\prime} \text { after extraction; } \\
\text { intermittent oozing } \\
\text { in the first } 24 \mathrm{~h} \text { ( } 3 \text { in } \\
\text { the control group } \\
\text { and } 2 \text { in the study } \\
\text { group). No serious } \\
\text { bleeding or } \\
\text { thromboembolic } \\
\text { event. }\end{array}$ \\
\hline $\begin{array}{c}\text { S. } \\
\text { Al-Mubarak, } \\
\text { M. Rass, A. } \\
\text { Alsuwyed, } \\
\text { A. } \\
\text { Alabulaaly, } \\
\text { S. Ciancio. }\end{array}$ & & $\begin{array}{l}\text { Warfarin } \\
(2-10 \mathrm{mg})\end{array}$ & $\begin{array}{l}\text { Yes, in } 2 \text { group, } \\
\text { Stop warfarin } 2 \\
\text { days prior to } \\
\text { surgery; } \\
\text { Resume } \\
\text { treatment } 12 \mathrm{~h} \\
\text { after. }\end{array}$ & $\begin{array}{l}\text { Antibiotic; } \\
\text { Painkiller, if } \\
\text { necessary. }\end{array}$ & $\begin{array}{c}\text { Teeth } \\
\text { extraction. }\end{array}$ & $\begin{array}{l}\text { Follow up } 7 \\
\text { days; phone } \\
\text { contact in case } \\
\text { of problems. }\end{array}$ & & 168 & $\begin{array}{c}\begin{array}{c}\text { Percentage of } \\
\text { bleeding in }\end{array} \\
\text { postoperative day 1: } \\
\text { 12,9\% (INR 1-2); } \\
18.9 \% \text { (INR 2-3); } \\
\text { higher (INR }>3 \text { ). No } \\
\text { intervention needed. } \\
\text { No thromboembolic } \\
\text { event. }\end{array}$ \\
\hline $\begin{array}{l}\text { A. Rocha, S. } \\
\text { Oliveira, A. } \\
\text { Souza, D. } \\
\text { Travassos, L. } \\
\text { Abreu, D. } \\
\text { Ribeiro, T. } \\
\text { Silva. }\end{array}$ & $\begin{array}{l}\text { Storage of the fluids } \\
\text { aspirated during the } \\
\text { surgical procedure } \\
\text { using a portable } \\
\text { vacuum pump. Score } \\
\text { 1: samples up to } 5 \\
\text { mL; score } 2: 6-10 \mathrm{~mL} \text {; } \\
\text { score } 3: 11-15 \mathrm{~mL} \text {, } \\
\text { and so on. } \\
\text { Post-operative } \\
\text { bleeding and wound } \\
\text { healing (satisfactory, } \\
\text { swelling/erythema, } \\
\text { or bone exposure). }\end{array}$ & $\begin{array}{l}\text { Warfarin } \\
\text { (5 mg). }\end{array}$ & No. & $\begin{array}{c}\text { Antibiotic } \\
\text { prophylaxis, if } \\
\text { necessary; } \\
\text { Paracetamol. }\end{array}$ & $\begin{array}{c}\text { Teeth } \\
\text { extraction. }\end{array}$ & $\begin{array}{c}60 \text { ' after } \\
\text { surgery; } \\
\text { Follow up } 7 \\
\text { days; phone } \\
\text { contact in case } \\
\text { of problems. }\end{array}$ & $\begin{array}{l}3.0 \text { nylon sutures; } \\
\text { gauze compression for } \\
20^{\prime} \text {. In case of } \\
\text { immediate bleeding: } \\
\text { absorbable gelatin } \\
\text { sponge, tranexamic } \\
\text { acid and/or new } \\
\text { sutures. }\end{array}$ & 138 & $\begin{array}{c}\text { Bleeding } \\
\text { complications: } 7 \text { in } \\
\text { anticoagulant } \\
\text { group; } 4 \text { in control } \\
\text { group. No } \\
\text { post-operative late } \\
\text { bleeds requiring } \\
\text { hospitalization } \\
\text { and /or blood } \\
\text { transfusions (only } \\
\text { haemostatic } \\
\text { measures). }\end{array}$ \\
\hline
\end{tabular}


Table 1. Cont.

\begin{tabular}{|c|c|c|c|c|c|c|c|c|c|}
\hline Author & $\begin{array}{l}\text { Description of } \\
\text { Primary Outcome }\end{array}$ & Anticoagulant & $\begin{array}{l}\text { Sospension of } \\
\text { Anticoagulant }\end{array}$ & Other Drugs & $\begin{array}{l}\text { Type of Oral } \\
\text { Surgery } \\
\text { Procedure }\end{array}$ & $\begin{array}{l}\text { Monitoring } \\
\text { Time and } \\
\text { Follow Up }\end{array}$ & $\begin{array}{l}\text { Method of Bleeding } \\
\text { Control }\end{array}$ & $\begin{array}{l}\text { Number of } \\
\text { Partecipants }\end{array}$ & $\begin{array}{c}\text { Number and Type } \\
\text { of Bleeding } \\
\text { Events }\end{array}$ \\
\hline $\begin{array}{l}\text { E. Soares, F. } \\
\text { Costa, T. } \\
\text { Bezerra, C. } \\
\text { Nogueira, P. } \\
\text { Silva, S. } \\
\text { Batista, F. } \\
\text { Sousa, C. } \\
\text { Fonteles. }\end{array}$ & $\begin{array}{l}\text { Postoperative } \\
\text { hemorrhage: } \\
\text { bleeding that could } \\
\text { not be controlled by } \\
\text { gauze compression } \\
\text { during } 20^{\prime} \text {, requiring } \\
\text { medical intervention. }\end{array}$ & $\begin{array}{l}\text { Warfarin } \\
\text { (INR 2.1-3.1). }\end{array}$ & No. & $\begin{array}{l}\text { Antibiotic } \\
\text { prophilaxis, if } \\
\text { necessary; } \\
\text { Paraceta- } \\
\text { mol;Avois } \\
\text { aspirin and } \\
\text { anti- } \\
\text { inflammatory } \\
\text { drugs for } \\
10 \text { days. }\end{array}$ & $\begin{array}{l}\text { Teeth } \\
\text { extraction } \\
(>2) \text {. }\end{array}$ & $\begin{array}{l}\text { Follow up } 7 \\
\text { days; phone } \\
\text { contact by } \\
\text { operator (12 h; } \\
24 \text { h); by the } \\
\text { patients (if } \\
\text { persisting } \\
\text { bleeding even } \\
\text { after } 20 \text { min of } \\
\text { gauze } \\
\text { compression, } \\
\text { or pain, or } \\
\text { fever). }\end{array}$ & $\begin{array}{l}\text { If persistent bleeding: } \\
\text { inspection and } \\
\text { curettage of surgical } \\
\text { site; fibrin sponge; } X \\
\text { suture; } 8^{\prime} \text { compression } \\
\text { with gauze soaked in } \\
4.8 \% \text { tranexamic acid. }\end{array}$ & 65 & $\begin{array}{l}\text { Postsurgical } \\
\text { bleeding 3.6-7.1\% } \\
\text { (mean of 5.3\%) } \\
\text { with tranexamic } \\
\text { acid and fibrin } \\
\text { sponge; } 3.6 \% \text { with } \\
\text { gauze compression } \\
\text { alone. In total } 4 \\
\text { bleeding episodes }\end{array}$ \\
\hline $\begin{array}{l}\text { G.Sammartino, } \\
\text { G. Marenzi, } \\
\text { A. Miro, F. } \\
\text { Ungaro, A. } \\
\text { Nappi, J. } \\
\text { Sammartino, } \\
\text { F. Quaglia, C. } \\
\text { Mortellaro. }\end{array}$ & & $\begin{array}{l}\text { Warfarin } \\
\text { (INR 2-4); } \\
\text { single/dual } \\
\text { anticoagula- } \\
\text { tion } \\
\text { therapy. }\end{array}$ & $\begin{array}{l}\text { Yes, in control } \\
\text { group; Stop } \\
\text { some days } \\
\text { before (INR < 2); } \\
\text { No, in test } \\
\text { group (INR > } \\
29 .\end{array}$ & $\begin{array}{l}\text { Avoid } \\
\text { cephalosporins, } \\
\text { macrolides, } \\
\text { quinolones } \\
\text { (interfering } \\
\text { with the } \\
\text { coagulation; } \\
\text { Diclofenac. }\end{array}$ & $\begin{array}{l}\text { Teeth } \\
\text { extraction } \\
(>2) .\end{array}$ & $\begin{array}{l}\text { Follow up } 7 \\
\text { days; phone } \\
\text { contact in case } \\
\text { of problems. }\end{array}$ & & 84 & $\begin{array}{l}6 \text { hemorrhagic } \\
\text { complications } \\
\text { (7.2\%). } 4 \text { in the } \\
\text { control group (late } \\
\text { postoperative } \\
\text { hemorrhage, } 2-4 \\
\text { days after } \\
\text { extraction period, } \\
\text { excessive } \\
\text { coagulum; } 2 \text { in the } \\
\text { study group, } \\
\text { immediate } \\
\text { postoperative } \\
\text { hemorrhage). }\end{array}$ \\
\hline
\end{tabular}


Table 1. Cont.

\begin{tabular}{|c|c|c|c|c|c|c|c|c|c|}
\hline Author & $\begin{array}{l}\text { Description of } \\
\text { Primary Outcome }\end{array}$ & Anticoagulant & $\begin{array}{l}\text { Sospension of } \\
\text { Anticoagulant }\end{array}$ & Other Drugs & $\begin{array}{c}\text { Type of Oral } \\
\text { Surgery } \\
\text { Procedure }\end{array}$ & $\begin{array}{l}\text { Monitoring } \\
\text { Time and } \\
\text { Follow Up }\end{array}$ & $\begin{array}{l}\text { Method of Bleeding } \\
\text { Control }\end{array}$ & $\begin{array}{l}\text { Number of } \\
\text { Partecipants }\end{array}$ & $\begin{array}{l}\text { Number and Type } \\
\text { of Bleeding Events }\end{array}$ \\
\hline $\begin{array}{l}\text { S. Queiroz, } \\
\text { V. Silvestre, } \\
\text { R. Soares, G. } \\
\text { Campos, A. } \\
\text { Germano, J. } \\
\text { da Silva. }\end{array}$ & $\begin{array}{l}\text { 1- Absent bleeding } \\
\text { (no complaints), 2- } \\
\text { Little (bloodstained), } \\
\text { 3-Moderate (some } \\
\text { amount of blood in } \\
\text { the mouth), 4-Severe } \\
\text { (large amounts of } \\
\text { blood in the mouth). }\end{array}$ & $\begin{array}{l}\text { Warfarin } \\
\text { (mean } \\
\text { INR 2.4). }\end{array}$ & No. & $\begin{array}{l}\text { Analgesic: } \\
\text { dipirona or } \\
\text { paracetamol. } \\
\text { Antibiotic } \\
\text { prophylaxis if } \\
\text { necessary. }\end{array}$ & $\begin{array}{c}\text { Teeth } \\
\text { extraction } \\
\text { (without flap } \\
\text { elevation and } \\
\text { ostectomy). }\end{array}$ & $\begin{array}{c}\text { Follow up } \\
7 \text { days, with } \\
12 \mathrm{~h}, 24 \mathrm{~h} \text {, and } \\
7 \text { days } \\
\text { controls. }\end{array}$ & $\begin{array}{l}\text { Test group: gauze } \\
\text { compression (5'); } \\
\text { suture; Control group: } \\
\text { compression }\left(5^{\prime}\right) \text { with } \\
\text { gauze soaked in } \\
\text { tranexamic acid. For } \\
\text { postoperative bleeding, } \\
\text { the procedure was } \\
\text { repeated. }\end{array}$ & 37 & $\begin{array}{c}\text { Severe bleeding in } 3 \\
(8.1 \%) \text { cases, mild in } \\
19(51.4 \%) \text { and } \\
\text { absent in } 15 \text { (40.5\%) } \\
\text { cases. In the first } 12 \\
\text { h postoperatively, } \\
\text { hemorrhage was } \\
\text { moderate in } 20 \\
\text { cases }(54.1 \%) \text {, mild } \\
\text { in } 13(35.1 \%) \text {, and } \\
\text { absent in four } \\
(10.8 \%) \text {. On the } \\
\text { seventh } \\
\text { postoperative day, } \\
\text { hemorrhage was } \\
\text { absent in all cases; } \\
\text { Time to achieve } \\
\text { cessation of } \\
\text { bleeding: } 9.1 \text { ( } \pm 3.6) \\
\text { minutes; lower for } \\
\text { the study group. }\end{array}$ \\
\hline $\begin{array}{l}\text { F. Al-Belasy, } \\
\text { M. Amer. }\end{array}$ & & Warfarin. & No. & $\begin{array}{l}\text { Amoxicillin or } \\
\text { Azithromycin; } \\
\text { Paracetamol, } \\
\text { if necessary; } \\
\text { Avoid aspirin } \\
\text { and other } \\
\text { nonsteroidal } \\
\text { antiinflamma- } \\
\text { tory for } 10 \\
\text { days after } \\
\text { surgey. }\end{array}$ & $\begin{array}{c}\text { Teeth } \\
\text { extractions } \\
\text { (also with } \\
\text { alveolplasty } \\
\text { and } \\
\text { mucoperiostal } \\
\text { flap). }\end{array}$ & $\begin{array}{l}\text { Follow up } \\
10 \text { days; phone } \\
\text { contact in case } \\
\text { of problems. }\end{array}$ & $\begin{array}{l}\text { Test group: histoacryl } \\
\text { glue; interrupted } \\
\text { resorbable sutures; } \\
\text { Control and negative } \\
\text { groups: gelatin sponge } \\
\text { and multiple } \\
\text { interrupted resorbable } \\
\text { sutures. }\end{array}$ & 40 & $\begin{array}{l}5 \text { in control group, } \\
\text { postoperative } \\
\text { spontaneous } \\
\text { bleeding requiring } \\
\text { treatment. }\end{array}$ \\
\hline
\end{tabular}


Table 1. Cont

\begin{tabular}{|c|c|c|c|c|c|c|c|c|c|}
\hline Author & $\begin{array}{l}\text { Description of } \\
\text { Primary Outcome }\end{array}$ & Anticoagulant & $\begin{array}{l}\text { Sospension of } \\
\text { Anticoagulant }\end{array}$ & Other Drugs & $\begin{array}{c}\text { Type of Oral } \\
\text { Surgery } \\
\text { Procedure } \\
\end{array}$ & $\begin{array}{l}\text { Monitoring } \\
\text { Time and } \\
\text { Follow Up }\end{array}$ & $\begin{array}{l}\text { Method of Bleeding } \\
\text { Control }\end{array}$ & $\begin{array}{l}\text { Number of } \\
\text { Partecipants }\end{array}$ & $\begin{array}{l}\text { Number and Type of } \\
\text { Bleeding Events }\end{array}$ \\
\hline $\begin{array}{l}\text { E. Karsli, Ö. } \\
\text { Erdogan, E. } \\
\text { Esen, E. } \\
\text { Acartürk. }\end{array}$ & $\begin{array}{l}\text { Intraoperative } \\
\text { bleeding: weight of } \\
\text { gauze swabs used } \\
\text { before and after } \\
\text { tamponade } \\
\text { measured with a fine } \\
\text { electronic weight } \\
\text { measurement device; } \\
\text { Postoperative } \\
\text { bleeding: patients } \\
\text { count the number of } \\
\text { extra gauze swabs } \\
\text { used for bleeding } \\
\text { control during the } \\
\text { first } 48 \mathrm{~h} \text {. }\end{array}$ & Warfarin. & $\begin{array}{l}\text { Yes, in } 2 \text { groups; } \\
\text { Stop } 3 \text { days } \\
\text { before, then } \\
\text { heparin } \\
\text { bridging up to } \\
24 \text { h before (INR } \\
\text { <2); Resumed } \\
\text { heparin after } \\
\text { haemostasis and } \\
\text { warfarin } 48 \text { h } \\
\text { after } \\
\text { (hospitalised); } \\
\text { No, in test } \\
\text { group. }\end{array}$ & $\begin{array}{l}\text { Antibiotic; } \\
\text { Painkiller, if } \\
\text { necessary. }\end{array}$ & $\begin{array}{l}\text { Teeth } \\
\text { extraction. }\end{array}$ & $\begin{array}{l}20^{\prime} \text { after } \\
\text { surgery; } \\
\text { follow up } 7 \\
\text { days, with } 48 \\
\text { h control. }\end{array}$ & $\begin{array}{l}\text { Gauze compression; } \\
\text { oxycellulose } \\
\text { dressing; suture 3.0.; } \\
\text { gauze compression } \\
\text { for } 1 \mathrm{~h} \text {. }\end{array}$ & 40 & $\begin{array}{l}\text { Higher values of } \\
\text { Amount Of Bleeding } \\
\text { in continuation } \\
\text { warfarin group; Mean } \\
\text { amounts of bleeding } \\
\text { were } 2.500 \text { mg in } \\
\text { continuation warfarin } \\
\text { group; } 1.000 \text { mg in } \\
\text { stop warfarin group; } \\
\text { 1.288 mg in stop } \\
\text { warafarin and } \\
\text { unfractioned heparin; } \\
\text { 1.736 mg in healthy } \\
\text { group. No severe } \\
\text { postoperative } \\
\text { bleeding in any } \\
\text { patient; number of } \\
\text { used extra gauze } \\
\text { swabs did not differ } \\
\text { significantly among } \\
\text { groups. }\end{array}$ \\
\hline $\begin{array}{l}\text { A. Scarano, } \\
\text { B. Sinjari, G. } \\
\text { Murmura, E. } \\
\text { Mijiritsky, F. } \\
\text { Iaculli, C. } \\
\text { Mortellaro, } \\
\text { S. Tetè. }\end{array}$ & $\begin{array}{l}\text { Bleeding events } \\
\text { measured by the scar } \\
\text { tissue over the } \\
\text { sockets: incomplete } \\
\text { closure (poor } \\
\text { healing); solid cloth } \\
\text { over the socket (no } \\
\text { bleeding); cloth that } \\
\text { sheds and oozing } \\
\text { tissue (positive } \\
\text { bleeding). }\end{array}$ & Warfarin. & No. & $\begin{array}{l}\text { Antibiotic; } \\
\text { Painkiller, if } \\
\text { necessary. }\end{array}$ & $\begin{array}{l}\text { Teeth } \\
\text { extraction. }\end{array}$ & $\begin{array}{l}1 \mathrm{~h} \text { after } \\
\text { surgery; } \\
\text { follow up } 7 \\
\text { days, with day } \\
\text { 3, day } 5 \\
\text { controls. }\end{array}$ & $\begin{array}{l}\text { Control group: only } \\
\text { suture; Test group: } \\
\text { suture plus socket } \\
\text { filled with CaS in } \\
\text { layers. }\end{array}$ & 30 & $\begin{array}{c}\text { No bleeding in suture } \\
\text { group; some bleedig } \\
\text { in CaS group on day } \\
1 .\end{array}$ \\
\hline $\begin{array}{l}\text { B. Bajkin, S. } \\
\text { Selakoviü, S. } \\
\text { Mirkoviü, I. } \\
\text { Šarpev, A. } \\
\text { Tadiü, B. } \\
\text { Milekiü. }\end{array}$ & $\begin{array}{l}\text { Bleeding event if it is } \\
\text { not handle by the } \\
\text { patient by himself } \\
\text { (gauze pressure). } \\
\text { Immediate or } \\
\text { delayed. }\end{array}$ & Warfarin. & No. & $\begin{array}{l}\text { Antibiotic; } \\
\text { Painkiller, if } \\
\text { necessary. }\end{array}$ & $\begin{array}{l}\text { Teeth } \\
\text { extraction. }\end{array}$ & $\begin{array}{c}2 \mathrm{~h} \text { after } \\
\text { surgery; } \\
\text { follow up } 5 \\
\text { days, with day } \\
\text { 1, day } 3 \\
\text { controls. }\end{array}$ & $\begin{array}{l}\text { Suture; absorbable } \\
\text { gelatin sponge; } \\
\text { gauze pressure. }\end{array}$ & 90 & $\begin{array}{l}\text { Postoperative } \\
\text { immediate bleeding: } 1 \\
(3.3 \%) \text { in gelatine } \\
\text { sponge group A; } 2 \\
(6.7 \%) \text { in other } \\
\text { groups. }\end{array}$ \\
\hline
\end{tabular}


Table 1. Cont.

\begin{tabular}{|c|c|c|c|c|c|c|c|c|c|}
\hline Author & $\begin{array}{l}\text { Description of } \\
\text { Primary Outcome }\end{array}$ & Anticoagulant & $\begin{array}{l}\text { Sospension of } \\
\text { Anticoagulant }\end{array}$ & Other Drugs & $\begin{array}{l}\text { Type of Oral } \\
\text { Surgery } \\
\text { Procedure }\end{array}$ & $\begin{array}{l}\text { Monitoring } \\
\text { Time and } \\
\text { Follow Up }\end{array}$ & $\begin{array}{l}\text { Method of Bleeding } \\
\text { Control }\end{array}$ & $\begin{array}{l}\text { Number of } \\
\text { Partecipants }\end{array}$ & $\begin{array}{l}\text { Number and Type of } \\
\text { Bleeding Events }\end{array}$ \\
\hline $\begin{array}{l}\text { W. } \\
\text { Halfpenny, J. } \\
\text { Fraser, D. } \\
\text { Adlam. }\end{array}$ & $\begin{array}{c}\text { Immediate or } \\
\text { delayed. Severity } \\
\text { scale of } \\
\text { post-operative pain: } \\
\text { no pain, moderate } \\
\text { pain, severe pain. }\end{array}$ & Warfarin. & No. & $\begin{array}{l}\text { Antibiotic; } \\
\text { Painkiller, if } \\
\text { necessary. }\end{array}$ & $\begin{array}{l}\text { Teeth } \\
\text { extraction. }\end{array}$ & $\begin{array}{l}\text { Follow up } 7 \\
\text { days; phone } \\
\text { contact in case } \\
\text { of problems. }\end{array}$ & $\begin{array}{l}\text { Gauze compression; } \\
\text { resorbable } \\
\text { oxycellulose or fibrin } \\
\text { adhesive; suture. }\end{array}$ & 46 & $\begin{array}{l}\text { No immediate } \\
\text { postoperative } \\
\text { bleeding; } 1 \\
\text { postextraction } \\
\text { hemorrhage }(24 \mathrm{~h}) \\
\text { re-sutured in } \\
\text { oxycellulose group } \\
\text { and } 1 \text { in fibrin group; } \\
1 \text { hospitalizasione for } \\
\text { persistent intermittent } \\
\text { bleeding in fibrin } \\
\text { group; postoperative } \\
\text { pain more frequent in } \\
\text { oxycellulose group. }\end{array}$ \\
\hline $\begin{array}{l}\text { I. Evans, M. } \\
\text { Sayers, A. } \\
\text { Gibbons, G. } \\
\text { Price, H. } \\
\text { Snooks, A. } \\
\text { Sugar. }\end{array}$ & $\begin{array}{l}\text { Immediate or } \\
\text { delayed. Sought help } \\
\text { by phone is also a } \\
\text { bleeding event. }\end{array}$ & Warfarin. & $\begin{array}{l}\text { Yes, in control } \\
\text { group; Stop } 2 \\
\text { days before } \\
\text { surgery; No, in } \\
\text { test group. }\end{array}$ & $\begin{array}{l}\text { Antibiotic; } \\
\text { Painkiller, if } \\
\text { necessary. }\end{array}$ & $\begin{array}{l}\text { Teeth } \\
\text { extraction. }\end{array}$ & $\begin{array}{c}10^{\prime} \text { after } \\
\text { surgery; } \\
\text { Follow up } 7 \\
\text { days; phone } \\
\text { contact in case } \\
\text { of problems. }\end{array}$ & $\begin{array}{c}\text { Gauze compression for } \\
\text { 10'; socket packed with } \\
\text { oxycellulose dressing; } \\
\text { suture. }\end{array}$ & 109 & $\begin{array}{c}\text { Immediate } \\
\text { postoperative } \\
\text { bleeding: } 3 \text { in at and } 3 \\
\text { in ctr; delayed } \\
\text { postoperative } \\
\text { bleeding } 9 \text { in at and } 7 \\
\text { in ctr; rate of bleeding } \\
\text { complications: } 26 \% \text { at } \\
\text { and } 14 \% \text { ctr. }\end{array}$ \\
\hline $\begin{array}{l}\text { G. Carter, A. } \\
\text { Goss, J. } \\
\text { Lloyd, R. } \\
\text { Tocchetti. }\end{array}$ & $\begin{array}{l}\text { Event that can't be } \\
\text { controlled by biting } \\
\text { the gauze pad for } 20 \\
\text { min. Pain, edema, } \\
\text { haematoma. }\end{array}$ & Warfarin. & No. & $\begin{array}{l}\text { Antibiotic; } \\
\text { Painkiller, if } \\
\text { necessary. }\end{array}$ & $\begin{array}{l}\text { Teeth } \\
\text { extraction. }\end{array}$ & $\begin{array}{l}\text { Follow up } 7 \\
\text { days, with day } \\
\text { 1, day } 3 \\
\text { controls. }\end{array}$ & $\begin{array}{l}\text { Test group: tranexamic } \\
\text { acid rinse after } \\
\text { extraction; absorbable } \\
\text { oxidized cellulose } \\
\text { mesh placed in the } \\
\text { apical third; resorbable } \\
\text { suture. Control group: } \\
\text { Surgicel in the apical } \\
\text { third; application of } \\
\text { fibrin glue to } \\
\text { the socket walls, } \\
\text { suture and final } \\
\text { application of the glue. }\end{array}$ & 49 & $\begin{array}{c}\text { No bleeding } \\
\text { complications in TxA } \\
\text { group; } 2 \text { light } \\
\text { bleedings on day } 2 \text { in } \\
\text { fibrin glue group. } \\
\text { Both required } \\
\text { intervention. }\end{array}$ \\
\hline
\end{tabular}


Table 1. Cont.

\begin{tabular}{|c|c|c|c|c|c|c|c|c|c|}
\hline Author & $\begin{array}{l}\text { Description of } \\
\text { Primary Outcome }\end{array}$ & Anticoagulant & $\begin{array}{l}\text { Sospension of } \\
\text { Anticoagulant }\end{array}$ & Other Drugs & $\begin{array}{l}\text { Type of Oral } \\
\text { Surgery } \\
\text { Procedure }\end{array}$ & $\begin{array}{l}\text { Monitoring } \\
\text { Time and } \\
\text { Follow Up }\end{array}$ & $\begin{array}{l}\text { Method of Bleeding } \\
\text { Control }\end{array}$ & $\begin{array}{l}\text { Number of } \\
\text { Partecipants }\end{array}$ & $\begin{array}{l}\text { Number and Type of } \\
\text { Bleeding Events }\end{array}$ \\
\hline $\begin{array}{l}\text { G. Carter, A. } \\
\text { Goss. }\end{array}$ & $\begin{array}{l}\text { Immediate or } \\
\text { delayed. Occurence } \\
\text { of haematoma or } \\
\text { oedema. Pain. }\end{array}$ & Warfarin. & No. & $\begin{array}{l}\text { Antibiotic; } \\
\text { Painkiller, if } \\
\text { necessary. }\end{array}$ & $\begin{array}{l}\text { Teeth } \\
\text { extraction. }\end{array}$ & $\begin{array}{c}20 \text { ' after } \\
\text { surgery; } \\
\text { follow up } 7 \\
\text { days, with day } \\
\text { 1, day } 3 \\
\text { controls. }\end{array}$ & $\begin{array}{c}\text { Irrigation with } 4.8 \% \\
\text { tranexamic acid } \\
\text { mouthwash; } \\
\text { oxycellulose sponge } \\
\text { submerged in TA } \\
\text { placed in the socket; } \\
\text { resorbable suture; } \\
\text { gauze compression for } \\
20^{\prime} \text {. }\end{array}$ & 85 & $\begin{array}{l}\text { Similar low bleeding } \\
\text { rate; postoperative } \\
\text { bleeding: } 3 \text { (severe } \\
\text { periodontitis). }\end{array}$ \\
\hline $\begin{array}{l}\text { C. Bacci, M. } \\
\text { Maglione, L. } \\
\text { Favero, A. } \\
\text { Perini, R. Di } \\
\text { Lenarda, M. } \\
\text { Berengo, E. } \\
\text { Zanon. } \\
\end{array}$ & & Warfarin. & No (INR 1.8-4). & $\begin{array}{c}\text { Antibiotic } \\
\text { prophylaxis, if } \\
\text { necessary; } \\
\text { Paracetamol } \\
\text { or Ibuprofen if } \\
\text { necessary; } \\
\text { Avoid aspirin. }\end{array}$ & $\begin{array}{l}\text { Teeth } \\
\text { extraction. }\end{array}$ & $\begin{array}{l}\text { Follow up } 8 \\
\text { days with day } \\
3 \text { control. }\end{array}$ & $\begin{array}{l}\text { Fibrin sponges; silk } \\
\text { sutures; gauze } \\
\text { compression with } \\
\text { tranexamic acid for } \\
30^{\prime}-40^{\prime} \text {; ice bag for } 1 \mathrm{~h} \text {. }\end{array}$ & 898 & $\begin{array}{c}\text { Bleeding } \\
\text { complications: } 7 \text { in } \\
\text { anticoagulant group; } \\
4 \text { in control group; } \\
\text { local haemostatic } \\
\text { measures). }\end{array}$ \\
\hline $\begin{array}{l}\text { R. da Silva, } \\
\text { T. Gadelha, } \\
\text { R. Luiz, S. } \\
\text { Torres. }\end{array}$ & $\begin{array}{l}\text { 1-No bleeding, } \\
\text { 2-Mild (blood in the } \\
\text { saliva), 3-Moderate } \\
\text { (gauze compression), } \\
\text { 4-Severe (surgical } \\
\text { re-intervention } \\
\text { and/or hospital } \\
\text { admission). }\end{array}$ & $\begin{array}{l}\text { Warfarin; } \\
\text { with/not } \\
\text { Antiplatelet } \\
\text { agents. }\end{array}$ & No. & $\begin{array}{l}\text { Antibiotic } \\
\text { prophylaxis, if } \\
\text { necessary. }\end{array}$ & $\begin{array}{l}\text { Single tooth } \\
\text { extraction. }\end{array}$ & $\begin{array}{c}20 \text { ' after } \\
\text { surgery; } \\
\text { follow up } 7 \\
\text { days. } \\
7 \text { days postop- } \\
\text { eratively. }\end{array}$ & $\begin{array}{l}\text { Bidigital alveolar } \\
\text { compression with } \\
\text { sterile gauze for } 5^{\prime} \text { min; } \\
\text { intra-alveolar EACA in } \\
\text { test group. }\end{array}$ & 52 & $\begin{array}{l}1 \text { immediate bleeding } \\
\text { in test group; } 23 \text { late } \\
\text { bleeding (16.4\%): } 11 \\
(15.7 \%) \text { in test group } \\
\text { and } 12(17.1 \%) \text { in } \\
\text { control group. Of } \\
\text { these } 23: 18(78.3 \%) \\
\text { moderate(controlled } \\
\text { by the patient by } \\
\text { gauze pressure); } \\
5(21.7 \%) \text { required } \\
\text { re-intervention. }\end{array}$ \\
\hline
\end{tabular}


Table 2. Studies considering NOACs.

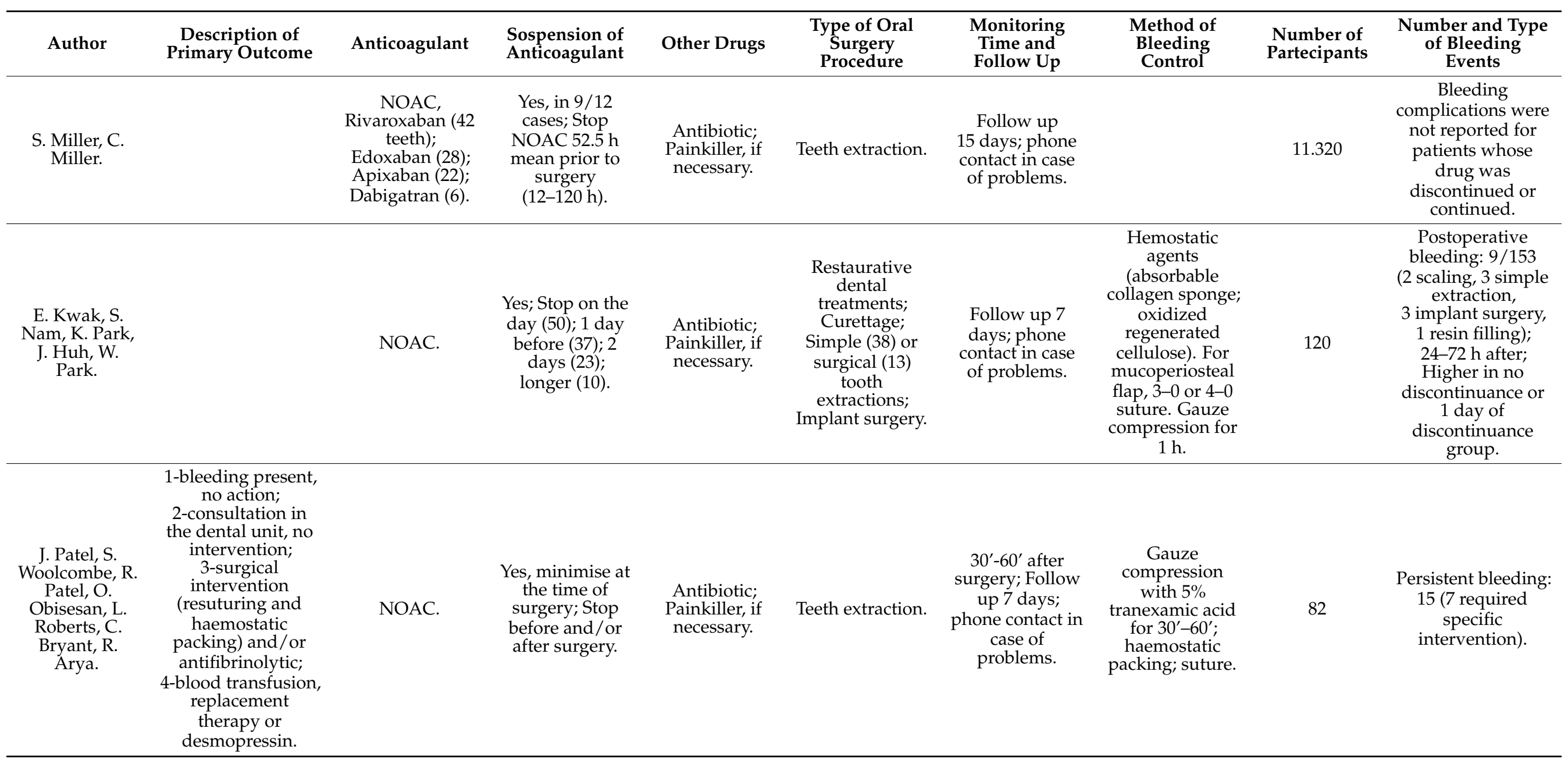


Table 2. Cont.

\begin{tabular}{|c|c|c|c|c|c|c|c|c|c|}
\hline Author & $\begin{array}{l}\text { Description of } \\
\text { Primary Outcome }\end{array}$ & Anticoagulant & $\begin{array}{l}\text { Sospension of } \\
\text { Anticoagulant }\end{array}$ & Other Drugs & $\begin{array}{l}\text { Type of Oral } \\
\text { Surgery } \\
\text { Procedure }\end{array}$ & $\begin{array}{l}\text { Monitoring } \\
\text { Time and } \\
\text { Follow Up }\end{array}$ & $\begin{array}{c}\text { Method of } \\
\text { Bleeding Control }\end{array}$ & $\begin{array}{c}\text { Number of } \\
\text { Parteci- } \\
\text { pants }\end{array}$ & $\begin{array}{c}\text { Number and Type } \\
\text { of Bleeding } \\
\text { Events }\end{array}$ \\
\hline $\begin{array}{l}\text { N. Cocero, M. } \\
\text { Basso, S. } \\
\text { Grosso, S. } \\
\text { Carossa. }\end{array}$ & $\begin{array}{l}\text { 1-Mild bleeding: } \\
\text { oozing; 2-Moderate: } \\
\text { not manageable by } \\
\text { the patient; } \\
\text { 3-Severe: not } \\
\text { manageable with } \\
\text { topical hemostatic } \\
\text { measures (systemic } \\
\text { therapy and/or } \\
\text { hospitalization). }\end{array}$ & $\begin{array}{c}\text { NOAC: } \\
\text { Dabigatran; } \\
\text { Apixaban; } \\
\text { Rivaroxaban. }\end{array}$ & $\begin{array}{c}\text { Yes, Stop NOAC } \\
4 \text { h before } \\
\text { surgery. }\end{array}$ & $\begin{array}{c}\text { Antibiotic } \\
\text { prophylaxis, if } \\
\text { necessary; } \\
\text { Paracetamol. }\end{array}$ & $\begin{array}{c}\text { Teeth } \\
\text { extractions }(<3 ; \\
\text { atraumatic } \\
\text { manner, no } \\
\text { mucoperiosteal } \\
\text { flap raised and } \\
\text { without rotary } \\
\text { instruments). }\end{array}$ & $\begin{array}{c}\text { Follow up } 7 \\
\text { days, with day } 1 \text {, } \\
\text { day } 3 \text { controls. }\end{array}$ & $\begin{array}{c}\text { Digital } \\
\text { mechanical } \\
\text { pressure and } \\
\text { topical agents } \\
\text { (resorbable gelatin } \\
\text { sponges). For } \\
\text { wide alveolar } \\
\text { sockets, sutures } \\
\text { 3-0 silk; dressings } \\
\text { with 5\% } \\
\text { tranexamic acid. } \\
\text { Moderate } \\
\text { bleeding: } \\
\text { reintervention } \\
\text { with removal of } \\
\text { necrotic clot and } \\
\text { new suture } \\
\text { (suspension of the } \\
\text { NOAC until } \\
\text { following } \\
\text { morning). }\end{array}$ & 100 & $\begin{array}{c}4 \text { bleeding } \\
\text { episodes (1 } \\
\text { moderate } 1 \mathrm{~h} \text { after } \\
\text { and } 3 \text { mild on day } \\
1 \text { ) in comorbidity } \\
\text { group; None in the } \\
\text { non-comorbidity } \\
\text { group; Overall } \\
\text { bleeding rate, } 4 \text { of } \\
100 \text { (4\%); } 0 / 36 \text { ( } 0 \% \text {; } \\
\text { without } \\
\text { comorbidities); } \\
4 / 64 \text { (6.25\%; with } \\
\text { comorbidities). }\end{array}$ \\
\hline $\begin{array}{l}\text { H. Hanken, A. } \\
\text { Gröbe, M. } \\
\text { Heiland, R. } \\
\text { Smeets, L. } \\
\text { Kluwe, J. } \\
\text { Wikner, R. } \\
\text { Koehnke, A. } \\
\text { Al-Dam, W. } \\
\text { Eichhorn. }\end{array}$ & & $\begin{array}{c}\text { Rivaroxaban (20 } \\
\text { mg/day) } \\
\text { plus/not aspirin } \\
\text { (100 mg/day). }\end{array}$ & No. & $\begin{array}{c}\text { Antibiotic } \\
\text { prophylaxis, if } \\
\text { necessary; } \\
\text { Ibuprofen for } \\
3 \text { days. }\end{array}$ & Teeth extraction. & $\begin{array}{l}\text { Follow up } 7 \\
\text { days; phone } \\
\text { contact in case } \\
\text { of problems. }\end{array}$ & $\begin{array}{l}\text { Collagen fleece; } \\
\text { suture. In case of } \\
\text { bleeding: } \\
\text { compression; } \\
\text { fibrin glue; new } \\
\text { suture. }\end{array}$ & & $\begin{array}{l}\text { All bleeding events } \\
\text { in the first week; } \\
\text { more bleeding } \\
\text { complications in } \\
\text { patients taking } \\
\text { rivaroxaban }(11.5 \% \\
\text { vs. } 0.7 \%) .\end{array}$ \\
\hline
\end{tabular}


Table 2. Cont

\begin{tabular}{|c|c|c|c|c|c|c|c|c|c|}
\hline Author & $\begin{array}{l}\text { Description of } \\
\text { Primary Outcome }\end{array}$ & Anticoagulant & $\begin{array}{l}\text { Sospension of } \\
\text { Anticoagulant }\end{array}$ & Other Drugs & $\begin{array}{l}\text { Type of Oral } \\
\text { Surgery } \\
\text { Procedure }\end{array}$ & $\begin{array}{l}\text { Monitoring } \\
\text { Time and } \\
\text { Follow Up }\end{array}$ & $\begin{array}{c}\text { Method of } \\
\text { Bleeding } \\
\text { Control }\end{array}$ & $\begin{array}{c}\text { Number of } \\
\text { Parteci- } \\
\text { pants }\end{array}$ & $\begin{array}{l}\text { Number and Type of } \\
\text { Bleeding Events }\end{array}$ \\
\hline $\begin{array}{l}\text { I. Miclotte, M. } \\
\text { Vanhaverbeke, } \\
\text { J. Agbaje, } \\
\text { P.Legrand, T. } \\
\text { Vanassche, P. } \\
\text { Verhamme, C. } \\
\text { Politis. }\end{array}$ & $\begin{array}{l}\text { Amount of bleeding } \\
\text { during the } \\
\text { extraction, scored } \\
\text { on a scale from } 1 \text { to } \\
5 \text { (1:no bleeding; } 5: \\
\text { continued bleeding } \\
\text { despite standard } \\
\text { measures). Early } \\
\text { (day 1) and delayed } \\
\text { (day 7) bleeding. }\end{array}$ & $\begin{array}{l}\text { Rivaroxaban } \\
\text { (69\%); } \\
\text { Dabigatran; } \\
\text { Apixaban. }\end{array}$ & $\begin{array}{l}\text { Yes; Skip only } \\
\text { the dose on the } \\
\text { morning; } \\
\text { Resumed } 4 \mathrm{~h} \\
\text { after. }\end{array}$ & $\begin{array}{l}\text { Antibiotic; } \\
\text { Painkiller, if } \\
\text { necessary. }\end{array}$ & $\begin{array}{c}\text { Teeth } \\
\text { extractions } \\
\text { (mean 2.6; } \\
\text { syndesmotome } \\
\text { followed by } \\
\text { forceps } \\
\text { extraction } \\
\text { with/without } \\
\text { osteotomy). }\end{array}$ & $\begin{array}{c}\text { Follow up } 7 \\
\text { days, with day } 1 \\
\text { control. }\end{array}$ & $\begin{array}{l}\text { Suture with } \\
\text { Vicryl }^{\circledR}{ }^{3}-0 .\end{array}$ & 52 & $\begin{array}{l}\text { No difference in the } \\
\text { procedural bleeding } \\
\text { score ( } 3.15 \text { in the } \\
\text { NOAC group versus } \\
2.92 \text { in the control } \\
\text { group); Early bleeding } \\
\text { events: } 5 \text { in both } \\
\text { groups; Delayed } \\
\text { bleeding: } 7 \text { in } \\
\text { anticoagulated group, } \\
0 \text { in } \\
\text { non-anticoagulated } \\
\text { group. } 12 \text { patients in } \\
\text { the NOAC group and } \\
5 \text { patients in } \\
\text { control group had a } \\
\text { bleeding event; } \\
\text { No major } \\
\text { bleeding events. }\end{array}$ \\
\hline
\end{tabular}


Table 3. Studies considering both VKAs and NOACs.

\begin{tabular}{|c|c|c|c|c|c|c|c|c|c|}
\hline Author & $\begin{array}{l}\text { Description of } \\
\text { Primary Outcome }\end{array}$ & Anticoagulant & $\begin{array}{l}\text { Sospension of } \\
\text { Anticoagulant }\end{array}$ & Other Drugs & $\begin{array}{c}\text { Type of Oral } \\
\text { Surgery Procedure }\end{array}$ & $\begin{array}{l}\text { Monitoring } \\
\text { Time and } \\
\text { Follow Up }\end{array}$ & $\begin{array}{l}\text { Method of } \\
\text { Bleeding } \\
\text { Control }\end{array}$ & $\begin{array}{l}\text { Number of } \\
\text { Partecipants }\end{array}$ & $\begin{array}{l}\text { Number and } \\
\text { Type of } \\
\text { Bleeding } \\
\text { Events }\end{array}$ \\
\hline $\begin{array}{l}\text { R. Rubino, R. } \\
\text { Dawson, R. } \\
\text { Kryscio, M. } \\
\text { Al-Sabbagh, C. } \\
\text { Miller. }\end{array}$ & & $\begin{array}{l}\text { Dabigatran; } \\
\text { Rivaroxaban; } \\
\text { Apixaban; } \\
\text { Edoxaban; } \\
\text { Warfarin; (other } \\
\text { antiplatelet } \\
\text { drugs). }\end{array}$ & $\begin{array}{l}\text { No, in } 99.6 \% \text { of } \\
\text { patients; Yes, in } \\
4 \text { patients } \\
\text { (1-5 days). }\end{array}$ & $\begin{array}{l}\text { Antibiotic; } \\
\text { Painkiller, if } \\
\text { necessary. }\end{array}$ & $\begin{array}{c}\text { Scaling and root } \\
\text { planing (484); } \\
\text { implant } \\
\text { placements (218); } \\
\text { Open flap } \\
\text { debridements (23); } \\
\text { Gingival grafts } \\
\text { (16); Sinus lift with } \\
\text { lateral window } \\
\text { (15); Other (71). }\end{array}$ & $\begin{array}{l}\text { Follow up } 7 \\
\text { days; phone } \\
\text { contact in case } \\
\text { of problems. }\end{array}$ & & 456 & $\begin{array}{l}\text { Postoperative } \\
\text { bleeding: } 3 \\
(0.35 \%) ; \\
\text { resolved with } \\
\text { local hemostatic } \\
\text { measures. }\end{array}$ \\
\hline $\begin{array}{l}\text { M. Miranda, L. } \\
\text { Martinez, R. } \\
\text { Franco, V. Forte, } \\
\text { A. Barlattani, P. } \\
\text { Bollero. }\end{array}$ & $\begin{array}{l}\text { (1)extra-alveolar } \\
\text { clots and bleeding } \\
\text { after more } 24 \mathrm{~h}, \\
\text { requiring } \\
\text { reoperation; (2) } \\
\text { bleeding < } 24 \mathrm{~h} \text {, } \\
\text { controlled by } \\
\text { gauze pressure; (3) } \\
\text { uncontrollable } \\
\text { bleeding } \\
\text { after } 24 \mathrm{~h}, \\
\text { requiring } \\
\text { reoperation; (4) } \\
\text { haematomas; pain. }\end{array}$ & $\begin{array}{l}\text { Dabigatran; } \\
\text { Rivaroxaban; } \\
\text { Apixaban; } \\
\text { Warfarin. }\end{array}$ & $\begin{array}{l}\text { Yes, in group } 2 \\
\text { (INR > 3); Stop } \\
\text { warfarin } \\
\text { Bridging with } \\
\text { heparin; Stop } \\
\text { heparin } 12 \mathrm{~h} \\
\text { before; } \\
\text { Resumed } 1 \text { day } \\
\text { after. No, in } \\
\text { NOAC group. }\end{array}$ & $\begin{array}{l}\text { Antibiotic; } \\
\text { Painkiller, if } \\
\text { necessary. }\end{array}$ & Teeth extraction. & $\begin{array}{l}60^{\prime} \text { after surgery; } \\
\text { follow up } 7 \\
\text { days, with day } 1, \\
3 \text { day } 3 \text { controls. }\end{array}$ & $\begin{array}{c}\text { Socket irrigation } \\
\text { with tranexamic } \\
\text { acid; gelatine } \\
\text { sponge; suture; } \\
\text { gauze } \\
\text { compression } \\
\text { with tranexamic } \\
\text { acid for } 15^{\prime} ; \\
\text { mouth rinses } \\
\text { with a } 10 \mathrm{~mL} \text { of } \\
5 \% \text { tranexamic } \\
\text { acid solution for } \\
2^{\prime}, 4 / \text { day, for } 7 \\
\text { days. }\end{array}$ & 50 & $\begin{array}{l}\text { Postoperative } \\
\text { bleeding: } 12 \\
\text { patients in } \\
\text { warfarin group; } \\
\text { no episodes } \\
\text { NOAC group. }\end{array}$ \\
\hline $\begin{array}{l}\text { H. Yoshikawa, } \\
\text { M. Yoshida, M. } \\
\text { Yasaka, H. } \\
\text { Yoshida, Y. } \\
\text { Murasato, D. } \\
\text { Fukunaga, A. } \\
\text { Shintani, Y. } \\
\text { Okada. }\end{array}$ & $\begin{array}{l}\text { Oozing or marked } \\
\text { haemorrhage that } \\
\text { could not be } \\
\text { stopped by gauze } \\
\text { compression, } \\
\text { requiring medical } \\
\text { intervention. }\end{array}$ & $\begin{array}{c}\text { NOAC (119: } 32 \\
\text { dabigatran, } 31 \\
\text { rivaroxaban, } 39 \\
\text { apixaban, 17 } \\
\text { edoxabaN); } \\
\text { Warfarin (248). }\end{array}$ & $\begin{array}{c}\text { No, extractions } \\
\text { 6-7 h after last } \\
\text { dose; Resumed } \\
\text { after } \\
\text { haemostasis. }\end{array}$ & $\begin{array}{l}\text { Antibiotic; } \\
\text { Painkiller, if } \\
\text { necessary. }\end{array}$ & $\begin{array}{l}\text { Teeth extraction: } \\
\text { nonsurgical } \\
\text { extraction; surgical } \\
\text { extraction } \\
\text { (mucoperiosteal } \\
\text { flap and/or } \\
\text { osteotomy); } \\
\text { impacted tooth } \\
\text { extraction. }\end{array}$ & $\begin{array}{l}\text { Follow up } 7 \\
\text { days; phone } \\
\text { contact in case } \\
\text { of problems. }\end{array}$ & $\begin{array}{c}\text { Resorbable } \\
\text { gelatin sponge; } \\
\text { 3-0 silk sutures; } \\
\text { gauze } \\
\text { compression for } \\
1 \mathrm{~h} \text {. }\end{array}$ & 367 & $\begin{array}{c}\text { Postoperative } \\
\text { bleeding: } 4 \\
(3.1 \%) \text { in NOAC } \\
\text { group; } 23(8.8 \%) \\
\text { in warfarin } \\
\text { group. }\end{array}$ \\
\hline
\end{tabular}


Table 3. Cont

\begin{tabular}{|c|c|c|c|c|c|c|c|c|c|}
\hline Author & $\begin{array}{c}\text { Description of } \\
\text { Primary Outcome }\end{array}$ & Anticoagulant & $\begin{array}{l}\text { Sospension of } \\
\text { Anticoagulant }\end{array}$ & Other Drugs & $\begin{array}{c}\text { Type of Oral } \\
\text { Surgery Procedure }\end{array}$ & $\begin{array}{l}\text { Monitoring } \\
\text { Time and } \\
\text { Follow Up }\end{array}$ & $\begin{array}{l}\text { Method of } \\
\text { Bleeding } \\
\text { Control }\end{array}$ & $\begin{array}{l}\text { Number of } \\
\text { Partecipants }\end{array}$ & $\begin{array}{l}\text { Number and Type } \\
\text { of Bleeding Events }\end{array}$ \\
\hline $\begin{array}{c}\text { M. Caliskan, H. } \\
\text { Tükel, M. } \\
\text { Benlidayi, A. } \\
\text { Deniz. }\end{array}$ & $\begin{array}{c}\text { Intra-operative } \\
\text { bleeding measured } \\
\text { with } \\
\text { Karsli-Erdogan } \\
\text { method: avoid } \\
\text { contaminations to } \\
\text { the blood isolating } \\
\text { salivary ducts; } \\
\text { gauze swabs used } \\
\text { and then weighted. }\end{array}$ & $\begin{array}{l}\text { NOAC; } \\
\text { Warfarin. }\end{array}$ & No. & $\begin{array}{l}\text { Antibiotic; } \\
\text { Painkiller, if } \\
\text { necessary. }\end{array}$ & Teeth extraction. & $\begin{array}{c}20^{\prime} \text { after } \\
\text { surgery; follow } \\
\text { up } 7 \text { days, with } \\
\text { day } 2 \text { control. }\end{array}$ & $\begin{array}{c}\text { Gauze } \\
\text { compression for } \\
20 \text { min; oxidized } \\
\text { cellulose } \\
\text { dressing; suture } \\
\text { 3.0. }\end{array}$ & 86 & $\begin{array}{c}\text { The mean AOB in } \\
\text { warfarin group was } \\
\text { significantly higher } \\
\text { than the other } \\
\text { groups (1.388 mg; } \\
\text { 1.909 mg; } 3.673 \mathrm{mg} ; \\
1.593 \mathrm{mg}) ; \text { mild } \\
\text { bleeding on day 2: } 1 \text {, } \\
2,6,0 ; \text { moderate } \\
\text { bleeding on day 2: } 1 \text {, } \\
2,6,0 ; \text { mild bleeding } \\
\text { on day } 7: 0,1,1,0 ; \\
\text { moderate bleeding } \\
\text { on day } 7: 0,0,2,0 ; \\
\text { none hospitalization. }\end{array}$ \\
\hline $\begin{array}{c}\text { E. Lababidi, O. } \\
\text { Breik, J. Savage, } \\
\text { H. Engelbrecht, } \\
\text { R. Kumar, C. } \\
\text { Crossley. }\end{array}$ & & $\begin{array}{l}\text { NOACs, } \\
\text { warfarin }\end{array}$ & No. & $\begin{array}{l}\text { Antibiotic; } \\
\text { Painkiller, if } \\
\text { necessary. }\end{array}$ & Teeth extraction. & $\begin{array}{l}\text { Follow up } 7 \\
\text { days; phone } \\
\text { contact in case } \\
\text { of problems. }\end{array}$ & $\begin{array}{l}\text { Haemostatic } \\
\text { agent; suture. } \\
\text { Delayed } \\
\text { bleeding: } \\
\text { re-packing of } \\
\text { haemostatic } \\
\text { agent, gauze } \\
\text { pressure and } \\
\text { temporary } \\
\text { cessation of } \\
\text { rivaroxaban. }\end{array}$ & 93 & $\begin{array}{l}\text { Minor bleeding } \\
\text { events: } 4(10.5 \%) \text { in } \\
\text { continuation NOAC } \\
\text { group; } 9 \text { (15.3\%) in } \\
\text { warfarin group. No } \\
\text { bleeding in stop } \\
\text { NOAC group. }\end{array}$ \\
\hline $\begin{array}{l}\text { C. Mauprivez, R. } \\
\text { Khonsari, O. } \\
\text { Razouk, P. } \\
\text { Goudot, P. } \\
\text { Lesclous, V. } \\
\text { Descroix. }\end{array}$ & $\begin{array}{c}\text { Persistent oozing } \\
\text { or marked } \\
\text { hemorrhage over } \\
20 \text { min after tooth } \\
\text { extraction despite } \\
\text { local hemostasis; } \\
\text { bleeding episode } \\
\text { during the first } \\
7 \text { days. }\end{array}$ & $\begin{array}{l}\text { VKAs and } \\
\text { NOACs }\end{array}$ & $\begin{array}{l}\text { No, in the test } \\
\text { group; Yes, in } \\
\text { the control } \\
\text { group. }\end{array}$ & $\begin{array}{l}\text { Antibiotic; } \\
\text { Painkiller, if } \\
\text { necessary. }\end{array}$ & Teeth extraction. & $\begin{array}{l}30^{\prime}-40^{\prime} \text { after } \\
\text { surgery; follow } \\
\text { up } 8 \text { days, with } \\
\text { day } 3 \text { control. }\end{array}$ & $\begin{array}{c}\text { Oxidised } \\
\text { cellulose; } \\
\text { resorbable } \\
\text { suture; gauze } \\
\text { compression } \\
\text { with tranexamic } \\
\text { acid for } 30^{\prime}-40^{\prime} ; \\
\text { ice bag for } 1 \mathrm{~h} \text {. }\end{array}$ & 900 & $\begin{array}{l}\text { Bleeding events: } 7 \text { in } \\
\text { anticoagulant group } \\
\text { (6/7 late, } 2 \text { days } \\
\text { after; } 1 / 76 \text { days } \\
\text { after); } 4 \text { in the } \\
\text { control group ( } 2 \text { days } \\
\text { after); managed with } \\
\text { surgical treatment } \\
\text { and suture. }\end{array}$ \\
\hline
\end{tabular}


Table 3. Cont

\begin{tabular}{|c|c|c|c|c|c|c|c|c|c|}
\hline Author & $\begin{array}{c}\text { Description of } \\
\text { Primary Outcome }\end{array}$ & Anticoagulant & $\begin{array}{l}\text { Sospension of } \\
\text { Anticoagulant }\end{array}$ & Other Drugs & $\begin{array}{c}\text { Type of Oral } \\
\text { Surgery Procedure }\end{array}$ & $\begin{array}{l}\text { Monitoring } \\
\text { Time and } \\
\text { Follow Up }\end{array}$ & $\begin{array}{l}\text { Method of } \\
\text { Bleeding } \\
\text { Control }\end{array}$ & $\begin{array}{l}\text { Number of } \\
\text { Partecipants }\end{array}$ & $\begin{array}{l}\text { Number and Type } \\
\text { of Bleeding Events }\end{array}$ \\
\hline $\begin{array}{l}\text { R. Clemm, F. } \\
\text { Neukam, B. } \\
\text { Rusche, A. } \\
\text { Bauersachs, S. } \\
\text { Musazada, C. } \\
\text { Schmitt. }\end{array}$ & $\begin{array}{l}\text { Intraoperative } \\
\text { bleeding: low, } \\
\text { moderate, severe; } \\
\text { early bleeding (up } \\
\text { to } 24 \mathrm{~h} \text { after); late } \\
\text { bleeding }(>24 \mathrm{~h}) \text {. }\end{array}$ & $\begin{array}{c}\text { Warfarin; } \\
\text { Heparin (8 } \\
\text { bridging); } \\
\text { NOAC: } \\
\text { Dabigatran (6); } \\
\text { Rivaroxaban } \\
\text { (8); Apixaban } \\
\text { (2); } \\
\text { Antplatelet } \\
\text { (63, } 21 \text { plus an- } \\
\text { ticoagulation). }\end{array}$ & $\begin{array}{l}\text { Yes, in } 8 \\
\text { patients; } \\
\text { heparin } \\
\text { birdging. }\end{array}$ & $\begin{array}{l}\text { Antibiotic in } \\
\text { bone augmen- } \\
\text { tationand/or } \\
\text { an implant } \\
\text { insertion or } \\
\text { for antibiotic } \\
\text { prophilaxis. }\end{array}$ & $\begin{array}{l}\text { Dental implants; } \\
\text { Sinus floor } \\
\text { augmentation; } \\
\text { Vertical and/or } \\
\text { lateral } \\
\text { bonegrafting with } \\
\text { autologous bone } \\
\text { grafts. }\end{array}$ & $\begin{array}{l}\text { Follow up } 10 \\
\text { days, with day } \\
1 \text { control. }\end{array}$ & $\begin{array}{c}\text { Suture Vicryl } \\
\text { 5.0; in case of } \\
\text { bleeding: gauze } \\
\text { compression } \\
\text { with tranexamic } \\
\text { acid; additional } \\
\text { suture; revision. }\end{array}$ & 564 & $\begin{array}{c}7 \text { postoperative } \\
\text { bleedings in } 564 \\
\text { patients }(1.2 \%) .4 \text { in } \\
\text { anticoagulant } \\
\text { groups( } 3.4 \%), 3 \text { in } \\
\text { the non-AT group } \\
(0.6 \%) \text {. No } \\
\text { thromboembolic } \\
\text { complication. } 2 \\
\text { hospitalized (1 in } \\
\text { platelet aggregator } \\
\text { inhibitor group; } 1 \text { in } \\
\text { non-AT group). No } \\
\text { postoperative } \\
\text { bleeding in NOAC } \\
\text { group. }\end{array}$ \\
\hline $\begin{array}{l}\text { T. Yagyuu, M. } \\
\text { Kawakami, Y. } \\
\text { Ueyama, M. } \\
\text { Imada, M. } \\
\text { Kurihara, Y. } \\
\text { Matsusue, Y. } \\
\text { Imai, K. } \\
\text { Yamamoto, T. } \\
\text { Kirita. }\end{array}$ & $\begin{array}{l}\text { Bleeding that could } \\
\text { not be stopped by } \\
\text { gauze pressure, } \\
\text { requiring medical } \\
\text { treatment between } \\
\text { 30min and } 7 \text { days } \\
\text { after. }\end{array}$ & $\begin{array}{l}\text { Warfarin; } \\
\text { NOAC. }\end{array}$ & No. & $\begin{array}{l}\text { Antibiotic; } \\
\text { Painkiller, if } \\
\text { necessary. }\end{array}$ & Teeth extraction. & $\begin{array}{l}30^{\prime} \text { after } \\
\text { surgery; follow } \\
\text { up } 7 \text { days. }\end{array}$ & $\begin{array}{c}\text { Oxidised } \\
\text { cellulose or } \\
\text { gelatine sponge; } \\
\text { suture. }\end{array}$ & 543 & $\begin{array}{c}\text { Postextraction } \\
\text { bleeding: } 10.4 \% \\
\text { NOAC; } 12 \% \\
\text { Warfarin; } 0.9 \% \text { no } \\
\text { anticoagulant; local } \\
\text { hemostatic } \\
\text { measures. }\end{array}$ \\
\hline
\end{tabular}




\subsection{Results of Individual Sources of Evidence}

\subsubsection{VKAs}

Of the 19 studies including patients in therapy with VKAs, the discontinuation timing of the doses of the anticoagulant was not the same for each study. To overcome this drawback, two different groups were considered: 12 studies observed the occurrence of bleeding events when the therapy was not suspended, whereas seven studies considered at least one case/control group in which the therapy was suspended or bridged or modified.

1. Suspension of Anticoagulant

Sacco et al. [20] divided the patients in two groups. In group A, the INR was measured $72 \mathrm{~h}$ before the procedure. The dosages of acenocoumarol or warfarin were reduced until INR values between 1.5 and 2.0 were achieved on the day of the procedure, considering a target of 1.8. No local or general hemostatic measures were adopted during or after surgery. The INR was maintained at these levels for $24 \mathrm{~h}$ after surgery, and full anticoagulant dosage was resumed $48 \mathrm{~h}$ later. In group B, the INR was measured $72 \mathrm{~h}$ before the procedure and on the day of the surgery. Perioperative measures such as oxidized cellulose sponge, gelatin and tranexamic acid (TXA) mouthwash were used to control the bleeding. One-hundredand-thirty-one patients were included: 66 in group A and 65 in group B. Statistically, there was no significant difference between the two groups at the end of the study: no immediate bleeding; 10 episodes of mild post-surgical bleeding in group A and six in group B; in each case, the bleeding was well handled by local measures. Cannon et al. [4] included a total of 70 patients in warfarin therapy, undergoing dental extractions. The first 35 patients were enrolled in the control group and had their warfarin treatment stopped for two days prior to the procedure. The INR level on the day of the procedure had to be lower than 2.0; otherwise, the surgery was postponed to another day. The subsequent 35 patients were included in the study group, with no discontinuation of therapy. In the control group, local hemostatic measures and sutures were used on every socket, while in the study group none of those measures were used. None of the patients, in either the control or the study group, had any immediate post-operative bleeding. However, intermittent oozing was recorded during the first $24 \mathrm{~h}$ period in three patients in the control group and in two patients in the study group. This was easily controlled with local pressure gauze. Statistically, there was no difference in the outcome between the two groups. Al-Mubarak et al. [23] divided 168 patients in therapy with warfarin in four groups: (1) no suture, warfarin not discontinued; (2) no suture, warfarin interrupted; (3) socket suture, warfarin continued; and (4) socket suture, warfarin interrupted. They observed that (1) there was no significance difference in terms of bleeding events in the four groups, and (2) the INR levels dropped to 1.5 in the two groups with discontinuation of therapy. Furthermore, the bleeding events were more frequent in the two groups involving the socket sutured. Sammartino et al. [27] included 84 patients divided in two groups made by 31 (control group) and 53 (study group) subjects each. The control group stopped the anticoagulation regimen a few days before surgery, obtaining an INR $<2$. The study group maintained the regimen unchanged, achieving the control of hemostasis in the residual socket with absorbable swelling sponges loaded with TXA. There were only six bleeding episodes, and no significant difference between the groups. All the complications were handled successfully with a gauze moistened with TXA. Karsli et al. [28] formed four groups: continuation of warfarin, warfarin bridged with low-molecular-weight heparin (LMWH), warfarin bridged with unfractionated heparin and a control group of healthy individuals. Gauze weights used before and after blotting were measured with an electronic fine weight measurement device, and differences in weight before and after gauze blotting were interpreted as the amount of bleeding. No severe postoperative bleeding was detected in any patient, and the number of extra gauze swabs did not differ significantly among groups. Bajkin et al. [30] also studied the LMWH bridging approach in 105 patients compared to 109 subjects who continued with warfarin. In the latter group, warfarin was discontinued 3 to 4 days before the appointment, obtaining an INR level $<1.5$ the day 
of the intervention. In cases of INR above this level, the surgery was delayed. LMWH bridging started one day after the interruption of warfarin and stopped $12 \mathrm{~h}$ before surgery. All the few cases of bleeding were easily solved with local hemostatic measures, and no statistically significant difference was found between these two groups of patients. Already in 2002, Evans et al. [34] started to reconsider the discontinuation of warfarin in patients undergoing simple dental extractions, stating that the risk derived by the discontinuation could be much worse than bleeding events caused by the continued regimen which can be handled well by local measures.

\section{Continuation of Anticoagulant}

The focus of the studies reviewed in this section is the use of local hemostatic measures in patients that did not discontinue anticoagulant therapy. Exceptions are the papers of Rocha et al. [37] and Bajkin et al. [29] in which hemostatic measures were not evaluated.

Rocha et al. evaluated the pattern of bleeding in individuals under continued VKAs therapy and non-anticoagulated individuals undergoing dental extractions. Perioperative bleeding was quantified through the storage of the fluids aspirated during the surgical procedure using a portable vacuum pump to achieve a final score for bleeding. The values varied from 2 to 14, with lower scores indicating less perioperative bleeding. The few bleeding events showed no statistical difference between the two groups.

Bajkin et al. conducted a study assessing the effect of combined oral anticoagulantaspirin therapy on postoperative bleeding in patients undergoing tooth extractions. The three groups considered were (1) combined anticoagulant-aspirin therapy, (2) oral anticoagulant therapy and (3) aspirin therapy. Three cases of bleeding occurred in group 1, two cases in group 2 and no cases in group 3. All the events were easily solved by standard local measures.

Queiroz et al. [21] tried to provide the clinician with an effective method to control bleeding. They formed two groups: (a) socket compression with gauze moistened with saline solution and (b) socket compression with gauze moistened with TXA. The latter measure of local hemostasis in topical form with gauze compression and irrigation was shown to be more effective in reducing the time to attain immediate hemostasis and in preventing intermediate bleeding. Even before this, Al-Belasy et al. [24] studied another method of hemostasis control: the n-butyl-2-cyanoacrylate glue. The control group, not using such local measure, manifested a statistically significant number of bleeding events, while the study group showed reassuring results.

Da Silva et al. [25] in 2018 assessed a hemostatic agent that was not well documented in literature: epsilon-aminocaproic acid (EACA). EACA acts at the same level of the coagulation cascade as the TXA but is considered to be less efficient. The group formed with patients using EACA as local measure presented the same occurrence of late bleeding events as the control group using routine measures to control bleeding. Soares et al. [26] analyzed three groups: (1) gauze pad soaked in TXA and applied to the surgical alveolus for 8 min under biting pressure; (2) fibrin sponge packed into the surgical alveolus, biting down on dry gauze for $8 \mathrm{~min}$ for compression; and (3) dry gauze compression performed under biting pressure on the surgical alveolus for $8 \mathrm{~min}$ without the use of local hemostatic agents. There was no statistical difference between the three groups and between the three hemostatic measures, in terms of bleeding events. Scarano et al. [31] evaluated the use of calcium sulfate (CaS) as hemostatic agent. In group 1, the post-extractive socket was managed with obliterative suture only. In group 2, patients were treated with CaS placed into the sockets. Even if the occurrence of bleeding events was minimal, their frequency at day 1 postoperatively was significantly less in patients treated with CaS as compared with patients without. Bajkin et al. [32] also compared different local hemostatic solutions: (1) suture, (2) gelatin sponge into the socket without suturing and (3) neither of those two measures. Bleeding events in group 1, 2 and 3 were observed. All cases of bleeding were easily solved with local hemostatic measures. Halfpenny et al. [33] compared the use of a resorbable oxycellulose dressing with a fibrin adhesive. No discernible differences in terms of bleeding in the postoperative period was noted. Conversely, postoperative pain 
was reported more frequently in the group that used a resorbable oxycellulose dressing. TXA was also evaluated by Carter et al. [35], who compared it with an autologous fibrin glue preparation. Subjects in Group A were required to rinse with $10 \mathrm{~mL}$ of a $4.8 \%$ TXA solution 4 times a day for 7 days postoperatively, while patients in Group B received autologous fibrin glue intraoperatively. Overall, two patients from the autologous fibrin glue group presented postoperative bleeding and were found to have grossly elevated INR values on the day of bleeding. A second study by Carter et al. [36] proved the efficacy of the TXA, focusing on the mouthwash regimen to adopt in the postoperative days. Group A received postoperatively a 4.8\% TXA mouthwash for a 2-day period. Group $B$ received the same mouthwash and instructions postoperatively, to be continued for 5 days. Two patients in group A and one in group B had minor postoperative bleeds that required minor ambulatory intervention, but the difference was not statistically significant. Finally, Bacci et al. [39], in a large multicenter study, compared 451 patients in therapy with warfarin with 449 non-anticoagulated patients. The hemostatic measures used to control bleeding in the warfarin group were fibrin sponges, silk sutures and gauzes saturated with TXA. In the OAT group, seven events were observed, which were handled easily with local measures. In the control group, four cases occurred; however, the difference was not statistically significant.

\subsubsection{NOACs}

Cocero et al. [41] conducted a retrospective study considering 100 patients treated with direct oral anticoagulants. They divided the patients into two groups: 64 patients with comorbidities (diabetes, liver disease, renal insufficiency) and 36 without comorbidities. Four bleeding episodes were observed in the comorbidities group and none in the noncomorbidity group. In the comorbidities group, the factors triggering the episodes were the extraction of more than one tooth and the proximity of extracted teeth. The last dose of DOAC has been taken at least $4 \mathrm{~h}$ before the intervention.

Kwak et al. [13], in a retrospective study that included 120 patients (153 dental extractions), analyzed bleeding episodes in different procedures that were divided in two groups depending on the risk assessment: high and low bleeding risk. Low bleeding risk procedures consisted of impression-taking, crown preparation, root canal therapy and resin filling. High bleeding risk procedures were simple and complex tooth extractions, scaling and implant surgery. Bleeding occurred in nine out of 153 cases: two cases of scaling, three cases of simple extraction, three cases of first stage implant surgery and one case of resin filling. The number of cases was higher for no discontinuance of NOAC therapy or 1 day of discontinuance than for 2 or 3 days of discontinuance.

Miller et al. [42] conducted a retrospective evaluation involving patients who underwent a total of 98 dental extractions. Overall, 42 teeth were extracted with rivaroxaban, 28 with Edoxaban, 22 with apixaban and six with dabigatran. Single and multiple extractions were performed on patients without bleeding complications regardless continuation or discontinuation of the DOAC.

Patel et al. [44] conducted a retrospective study, in which 82 patients underwent 111 dental extractions. In 35 procedures, advice was given to omit the DOAC, either before or after treatment. There was no bleeding following the majority of procedures. Persistent bleeding followed 15 procedures, of which seven procedures required specific intervention such as re-suture.

Miclotte et al. [45] conducted a prospective case-control study in 26 patients undergoing dental extraction and treated with dabigatran, rivaroxaban or apixaban, and 26 matched controls not taking any antithrombotic drug. Patients were instructed to skip only the dose on the morning of the procedure. A procedural bleeding score was recorded, and early and delayed bleeding was assessed post-operatively at days 1 and 7 . There was no statistical difference in the procedural bleeding score or in early bleeding events ( 5 in both groups). However, delayed bleeding occurred more frequently in anticoagulated compared to non-anticoagulated patients. 
Hanken et al. [46] focused on the risk of bleeding in patients taking Rivaroxaban, the most widely used novel oral anticoagulant. It was a retrospective cohort study that included 52 oral procedures performed under continued oral anticoagulant therapy with rivaroxaban $20 \mathrm{mg} /$ day. Among them, two procedures were performed under continued dual therapy with aspirin $100 \mathrm{mg} /$ day added to the regimen. Postoperative bleeding events were compared with 285 oral procedures in patients without any anticoagulation/antiplatelet therapy. It resulted that there were more episodes of bleeding in the study group under rivaroxaban therapy $(15 \%)$ than in the control group $(0.7 \%)$. All the episodes occurred within the first week and were manageable with local hemostatic measures such as gauze compression and fibrin sponges.

\subsubsection{VKAs and NOACs}

In this group, the included studies considered patients in therapy with both VKAs and NOACs. The bleeding risk was compared together with the various local measures to control bleeding.

\section{Implant Therapy}

The study by Rubino et al. [47] actually did not consider patients undergoing tooth extractions, however the results deriving from implant and periodontal procedures have been reviewed as well. The authors considered patients under therapy with warfarin or novel anticoagulant, combined or not with antiplatelet drugs. Out of 867 procedures, the incidence of bleeding episodes was very low, being only three. The anticoagulant or antiplatelet therapy were not discontinued in any case, and the incidence of bleeding was $0.35 \%$.

Clemm et al. [50] also performed implant-related treatments including single or multiple implant insertions, implant exposures, sinus floor augmentation and vertical and/or lateral bone grafting with autologous bone grafts. Subjects in the test groups were treated with platelet aggregation inhibitors (PAIs), Vitamin K inhibitors, Vitamin K inhibitor withdrawal bridged with LMWH or NOACs. Patients in the control group were non-anticoagulated. There were seven postoperative bleedings in 564 patients, four in the test group, and three in the control group. In the NOAC group, no episodes were observed. All bleeding events were easily controlled with local hemostatic measures.

\section{Dental Extractions}

Yoshikawa et al. [48] conducted a prospective observational study that included 367 patients undergoing tooth extraction, 119 receiving NOACs and 248 receiving warfarin. All extractions in NOAC patients were performed 6-7 h after therapy in consideration of the blood half-life under continued antithrombotic treatment. Postoperative bleeding episodes occurred in four cases in the NOAC group and in 23 cases in the warfarin group. However, note that in the warfarin group, more complex surgical extractions were performed. Therefore, the difference in terms of bleeding events was not considered statistically significant. All cases of postoperative bleeding in the warfarin group were managed by local hemostatic measures such as compression with gauze, without stopping the anticoagulant therapy. A total of four bleeding episodes were recorded in the NOAC group: two were managed with local measures, while the other cases required the discontinuation of NOAC for one dose, in order to obtain a standard level of coagulation.

Lababidi et al. [43] conducted a retrospective controlled cohort study. All patients underwent dental extractions and three groups were identified: 29 patients on chronic NOAC therapy, 14 patients under NOAC therapy who discontinued the drug and a control group of 50 patients on chronic warfarin therapy without peri-procedural cessation. The incidence, severity and timing of bleeding events were recorded for each group. In the 53 procedures conducted within the NOAC group, 15 were done following perioperative cessation of the medication under physician advice, with large variability in the timing of suspension, ranging from 1 to 14 days. Four bleeding events were reported in the non-ceased NOAC group, two of them requiring reintervention. No bleeding events were 
reported in the ceased NOAC group. Within the warfarin group, there were nine episodes, with five requiring reintervention. There was no statistically significant difference between the three groups.

Caliskan et al. [49], in a randomized controlled trial, considered four different groups: patients taking direct thrombin inhibitors, patients taking factor Xa inhibitors, patients taking warfarin and a drug-free control group. A total of 84 patients underwent simple tooth extractions. The number of patients showing mild and moderate bleeding was significantly higher in warfarin group compared to other groups, and also the amount of bleeding in warfarin group was apparently higher.

Mauprivez et al. [38], in a prospective observational study, examined 51 patients who were treated with oral anticoagulants and required dental extractions. They were divided into two groups: 31 patients receiving NOAC and 20 control patients taking VKA with an INR between 2.0 and 3.0. In both groups, extractions were performed under continued $\mathrm{OAT}$, and the same local hemostatic measures were applied. In general, five patients taking NOACs showed seven bleeding episodes, whereas four patients treated with VKAs had five bleeding episodes during the postoperative follow-up period. The difference in the number of bleeding events between the two groups was not statistically significant. Only one episode required revision of the wound, application of fibrin glue and re-suturing.

Miranda et al. [51] performed a randomized clinical trial dividing 50 patients in two groups. Group A consisted of 12 patients treated with NOACs, while Group B consisted of 38 patients treated with Warfarin bridged with $\mathrm{LWMH}$, due to an INR value $>3$. The NOAC was not interrupted but the appointments were scheduled after 12 or $24 \mathrm{~h}$ from the last dose, so that the surgical procedures were performed with the lowest plasmatic concentration of the drug. Overall, 27\% cases in Group B showed an increased intraoperative bleeding, resulting in a reduction of the visibility of the operative field and higher difficulty in surgical procedures. This event did not occur in Group A. In 15.78\% of cases in Group B, widespread bleeding episodes required re-intervention, consisting in changing the systemic therapy with heparin and applying additional sutures at the surgical site. In Group A, a good management of the hemostasis was obtained, without intra- and post-operative bleeding complications.

Yagyuu et al. [52], in a recent retrospective cohort study, evaluated the primary outcome of bleeding after extractions. A total of 1196 tooth extractions in 541 patients fulfilled the inclusion criteria, with 72 extractions involving NOACs, 100 extractions involving VKAs and 1024 extractions involving controls with no anticoagulants. The incidences of postextractive bleeding per tooth for the NOAC, VKA and no anticoagulant extractions were $10.4 \%, 12.0 \%$ and $0.9 \%$, respectively.

\section{Discussion}

In oral surgery, excessive bleeding is a negative factor that may compromise the surgical procedure itself and increase the post-operative morbidity and discomfort for the patient. In this regard, a strict preoperative evaluation of the bleeding risk and clear protocols on how to handle bleeding events may contribute to minimize the complications experienced by both professionals and patients.

The aim of the present review was to explore the existing literature related to the management of patients in therapy with anticoagulant drugs undergoing dental surgery. Most of the studies that we considered evaluated patients scheduled for dental extractions (one of the most daily and standard procedure), while only a few considered other procedures such as implant placement (single or multiple), implant exposures, sinus floor augmentation and bone grafting with autologous bone. The primary outcome was the evaluation of the post-operative bleeding. Additional parameters were also investigated, including the suspension of the anticoagulant regimen, the type of anticoagulant therapy and the local measures adopted to contain bleeding. A scoping review might be considered the best method of research for this topic due to the fact that we are not answering a specific question (like the systematic review approach), but we are trying to summarize a 
much broader field of interrogatives [15]. Three main typologies of studies were identified: studies including patients in warfarin therapy, studies treating patients in NOAC therapy and studies that compared the two categories.

The approaches commonly adopted in case of anticoagulated patients during dental extractions varied from total discontinuation of OAT to the continuation of therapeutic levels of anticoagulation. Increasing numbers of authors have stated that dental extractions in anticoagulated patients can be successful without altering the therapy regimen, laying emphasis on local hemostatic measures [53]. The constant monitoring of INR values in these patients is crucial in the pre-surgical planning. On the basis of the individual response of each patient, the accidental drop of INR values to subtherapeutic levels can often occur after discontinuation of OAT, and this could represent a risk for patients without additional prophylaxis $[5,23]$.

The studies that suspended warfarin therapy aimed to obtain an INR level below 2.0 on the day of the intervention. There is no agreement between clinicians about the suspension regimen $[4,20,23]$. Bringing the INR values to the target level could result in an increased risk of severe consequences such as thromboembolic events that outweighs the risk of bleeding episodes during the extraction, as these episodes can be easily managed with local measures [27]. An important conclusion that can be drawn is that dental surgery can be safely conducted if INR levels are maintained between 1 and 4, controlling bleeding with the aid of local hemostatic agents.

As resulted in the present review, studies reporting on VKAs still represent the majority of literature with respect to anticoagulant drugs in dental patients.

Given the uncertainty about the ideal timing of drug suspension, an alternative strategy was often represented by bridging with LMWH. VKAs have a $40 \mathrm{~h}$ half-life. Thus, almost two days with a minimal blood concentration of drug may be dangerous and lead to severe thromboembolic consequences. It was therefore believed that a periprocedural heparin bridging strategy compared with no bridging would minimize the period of time without anticoagulation and thus reduce thromboembolic events at the expense of an acceptable increase in bleeding rates. This conceptual framework led to the generation of explicit protocols for periprocedural heparin bridging [54]. In this context, heparin bridging may be considered not useful anymore, because the fast onset time of the new anticoagulants brings the non-anticoagulation period from 2-3 days to much more manageable few hours.

Many studies considered the continuation of warfarin without cessation nor bridging. As different studies showed no differences between discontinuing and non-discontinuing warfarin in terms of bleeding events, the best solution is to continue anticoagulant therapy with VKAs. Once it is clear that non-discontinuation of warfarin may be considered the best approach, local hemostatic measures become absolutely necessary to control intra-surgical and post-operative bleeding.

Novel oral anticoagulants have proven to be safe and effective, offering a series of advantages including rapid action, no need for constant monitoring, few drug and food interactions, and a broad therapeutic margin. With such pharmacological advantages, it is not surprising that the number of such patients on NOACs being addressed by dental practitioners is increasing at a fast rate. Although in the present review there are still more studies on VKAs than NOACs, in the next few years the trend will probably reverse.

In 2018, Fortier et al. [55] reviewed the literature and concluded that, with the exception of a new reversal agent to direct thrombin inhibitors such as dabigatran, the lack of a specific antidote for factor Xa inhibitors makes the establishment of management protocols very difficult. Thus, caution should be taken when treating patients in therapy with NOACs until precise guidelines will be made available.

The recommended interruption of NOAC administration varies depending on the class of anticoagulant. The dental clinician should therefore become familiar with each of these time frames to inform the patient. Note that, if NOAC administration is interrupted pre-operatively, then NOAC re-administration should begin post-operatively following 
hemostasis [55]. In this matter, the discontinuation of NOACs was not an important factor in determining bleeding events in the studies considered in the present review $[13,42,44]$.

A relevant advantage of these molecules is the possibility to plan the intervention based on the last dose of NOAC taken, in order to minimize the intraoperative bleeding without altering the anticoagulant regimen. Different studies adopted this method, so that the procedure was scheduled several hours from the latest dose or just before the subsequent dose of NOAC. Interestingly, in each study assessed in the present review, this approach resulted in an excellent view of the surgical field [41,48,51]. Although this may be considered a valid approach, there is no conclusive evidence that recommends scheduling the appointment based on the last dose of NOAC. In this respect, Brennan et al. recently suggested that there is no need to adjust DOAC dosing prior to dental extractions, nor is there a need to time the dental extractions around DOAC doses [56].

Many different hemostatic methods have been proposed and used over the last decades. In the present review various local measures were identified, such as compression with gauze moistened with saline solution, dry gauze compression, TXA in the form of moistened gauzes and postoperative mouthwash, fibrin and gelatin sponges, autologous fibrin glue, resorbable oxycellulose dressing, N-butyl-2-cyanoacrilate glue (NBCA), epsilon-aminocaproic acid (EACA) and calcium sulfate (CaS) amongst others. Suture is not necessary if the socket is well represented in terms of well-represented bone walls and restraining defect. Note in this matter that some authors observed more bleeding events in sutured alveoli rather than in alveolar sockets left to heal spontaneously [23].

EACA affects the fibrinolytic system at several points. It acts primarily as an inhibitor of plasminogen activation, and only at high concentration $\left(5 \times 10^{\wedge} 2 \mathrm{M}\right)$ is able to inhibit plasmin directly. EACA is not as effective and safe as the TXA, which is 10 times more potent, but its action can be compared with common local measures such as gauze compression and fibrin sponges. [26] In fact, in the study conducted by Da Silva et al. [25], out of 140 patients, 70 in the study group using EACA and 70 in the control group using local pressure and other local measures, the authors reported a comparable incidence of late bleeding between the two groups, being $15.7 \%$ and $17.1 \%$, respectively. Considerable uncertainty thus remains about the efficacy and safety of EACA.

$\mathrm{CaS}$ has been widely employed throughout the years in different applications. It has been used in bone regeneration as a graft material and graft binder/extender and as a barrier in guided tissue regeneration. It has been shown that tissue often migrates over CaS if primary closure cannot be achieved [57]. Scarano et al. [31] exploited this aspect by placing $\mathrm{CaS}$ into the post-extractive sockets. Bleeding frequency at the first postoperative day was significantly lower in patients treated with CaS compared to patients CaS-free. No statistically significance differences in bleeding were noted after some days, meaning that CaS could be used to obtain a better hemostasis in the early stages of healing.

NBCA-based tissue adhesive was employed as a non-suture method to seal wounds in oral surgery. Al Belasy et al. [24] and previous studies [58] confirmed the efficacy of this local hemostatic. Al Belasy compared patients treated with Hystoacril glue placed in the socket right after the extraction (three different layers, allowing $30 \mathrm{~s}$ of time between applications) with patients treated with ordinary local measures such as gelatine sponges. Both the groups obtained an adequate hemostasis after the extractions, but it is remarkable how in the NBCA group the hemostasis was immediate, while it took between 10 and $20 \mathrm{~min}$ in the ordinary group. NBCA proved its efficacy also in terms of late bleeding, as no episodes occurred in this group in contrast to the five late episodes encountered in the ordinary group.

The most widely used hemostatic protocol to date is compression and mouthwash rinses with TXA $[20,21,25,27,33,35,36,39]$. The different formulations of TXA do not seem to affect its effectiveness. Indeed, although mouthwash is the most reported formulation, it may also be used in dressings with gauze, for local irrigation $(250 \mathrm{mg} / 5 \mathrm{~mL}$ solution), as a suspension of a tablet $(250 \mathrm{mg})$ crushed with saline or local anesthetics and used in gauze or even directly on the wound. TXA is a synthetic derivative of the amino acid lysine 
and exerts its antifibrinolytic effect through reversible blockade of lysine binding sites on plasminogen molecules. Although plasmin can still be formed under these circumstances, it is unable to bind to and degrade fibrin. [59] In the present review, the most prescribed concentration of TXA mouthwash was $4.8 \%$ and the postoperative regimen ranged from 2 to 5 up to 7 days. All these formulations resulted effective in containing bleeding episodes.

Even if no specific studies have been found in this respect, almost all authors use gauze compression as the first hemostatic measure. Moreover, TXA has been widely studied indirectly as it is often used as a reference local measure in control groups in the comparison with other hemostatic agents. Hemostasis by compression was obtained in different modalities: the gauze can be dry, it can be moistened with saline solution or it can be moistened with hemostatic agents like TXA. It is the authors' opinion, based on the everyday clinical experience, that using dry gauze can dislodge the blood clot, so it is advisable to always wet these devices. In a study, the time required to attain hemostasis was higher when using gauzes moistened with saline compression compared to TXA, and this difference was statistically significant. However, despite the slightly delayed effect in comparison with TXA, compression with gauzes appears to be a safe method in controlling postoperative bleeding in ambulatory dental surgery, particularly when used preventively in patients taking oral anticoagulants and also when used as secondary measures for the control of delayed bleeding [21].

\section{Conclusions}

Anticoagulant therapy with warfarin, whether continued, stopped or bridged a few days before surgery with heparin therapy, seems to be no longer preferred in clinical settings. This is mainly related of the difficult management of vitamin $\mathrm{K}$ antagonists, their interaction with numerous drugs and foods and their constant need to be monitored for effectiveness and blood concentration. The trend is now directed toward the prescription of direct oral anticoagulants, which have far fewer interactions and better perioperative management. The four new oral anticoagulants, namely dabigatran, rivaroxaban, apixaban and edoxaban evaluated in the present review showed effectiveness and safety, despite some differences in indications by to age groups. In case of extractive surgery, one of the most widely adopted protocols to reduce the risk of bleeding is to perform surgery as far away as possible from the last administration, with no drug suspension. All bleeding episodes that occurred in the studies reviewed herein were easily controlled using conventional hemostasis techniques. Many different hemostatic agents have been investigated, including fibrin and gelatin sponges, autologous fibrin glue, resorbable oxycellulose dressing, N-butyl-2-cyanoacrylate glue, epsilon-aminocaproic acid, calcium sulphate and suturing. The most effective, reliable and widely used in the literature is apparently compression with gauze moistened with saline solution or tranexamic acid, which can also be used effectively in the form of mouthwashes. As literature is extremely lacking in providing evidence on the management of new oral anticoagulants in dental surgery, further clinical studies are needed to confirm these initial conclusions.

Author Contributions: Conceptualization, M.M. and L.C.; methodology, P.P.P.; validation, M.B., and C.M.; formal analysis, A.P.; investigation, A.P.; data curation, P.P.P.; writing-original draft preparation, A.P.; writing-review and editing, M.M. and P.P.P.; supervision, M.B.; project administration, L.C. and C.M. All authors have read and agreed to the published version of the manuscript.

Funding: This research received no external funding.

Institutional Review Board Statement: This article does not contain any studies with human participants or animals performed by any of the authors.

Informed Consent Statement: For this type of study, formal consent is not required.

Conflicts of Interest: The authors declare no conflict of interest. 


\begin{abstract}
Abbreviations
PT (prothrombin time), INR (international normalized ratio), VKAs (vitamin K antagonists), NOAC (new oral anticoagulants), DOAC (direct oral anticoagulants), NBCA (N-butyl-2-cyanoacrilate), CaS (calcium sulfate), EACA (epsilon amino caproic acid), TXA (tranexamic acid).
\end{abstract}

\title{
References
}

1. Devlin, T.D. Textbook of Biochemistry with Clinical Correlations; Wiley: New York, NY, USA, 2012.

2. Chalmers, E.A.; Bonduel, M.M. Antithrombotic therapy in children. Prog. Pediatr. Cardiol. 2005, 21, 31-43. [CrossRef]

3. Salem, D.N.; Stein, P.D.; Al-Ahmad, A.; Horstkottr, D.; Miller, N.; Pauker, S.G. Antithrombotic therapy in valvular heart diseaseNative and prosthetic: The Seventh ACCP Conference on Antithrombotic and Thrombolytic Therapy. Chest 2004, 126, 457S-482S. [CrossRef]

4. Cannon, P.D.; Dharmar, V.T. Minor oral surgical procedures in patients on oral anticoagulants-A controlled study. Aust. Dent. J. 2003, 48, 115-118. [CrossRef] [PubMed]

5. Devani, P.; Lavery, K.M.; Howell, C.J.T. Dental extractions in patients on warfarin: Is alteration of anticoagulant regime necessary? Br. J. Oral Maxillofac. Surg. 1998, 36, 107-111. [CrossRef]

6. Katzung, P.; Master, T. Farmacologia Generale e Clinica; Piccin Nuova Libraria: Padova, Italy, 2011.

7. Bajkin, B.V.; Urosevic, I.M.; Stankov, K.M.; Petrovic, B.B.; Bajkin, I.A. Dental extractions and risk of bleeding in patients taking single and dual antiplatelet treatment. Br. J. Oral Maxillofac. Surg. 2015, 53, 39-43. [CrossRef] [PubMed]

8. Febbo, A.; Cheng, A.; Goss, A.; Stein, M.; Sambrook, P. Postoperative bleeding following dental extractions in warfarinised patients. Int. J. Oral Maxillofac. Surg. 2015, 44, e207. [CrossRef]

9. Sandvik, L.; Erikssen, J.; Thaulow, E.; Erikssen, G. The New England Journal of Medicine Downloaded from nejm.org at Mcgill University Library on November 30, 2015. Phys Fit as a Predict Mortal Men. 1993, 328, 2010-2013.

10. Johnson-Leong, C.; Rada, R.E. The use of low-molecular-weight heparins in outpatient oral surgery for patients receiving anticoagulation therapy. J. Am. Dent. Assoc. 2002, 133, 1083-1087. [CrossRef] [PubMed]

11. Johnson, W.T.; Leary, J.M. Management of dental patients with bleeding disorders: Review and update. Oral Surg. Oral Med. Oral Pathol. 1988, 66, 297-303. [CrossRef]

12. Ciulla, M.M.; Vivona, P. Novel oral anticoagulants: A practical guide for dentists. ItalIan J. Dent. Med. $2018,3,7-11$.

13. Kwak, E.J.; Nam, S.; Park, K.M.; Kim, S.Y.; Huh, J.; Park, W. Bleeding related to dental treatment in patients taking novel oral anticoagulants (NOACs): A retrospective study. Clin. Oral Investig. 2019, 23, 477-484. [CrossRef]

14. Chahine, J.; Khoudary, M.N.; Nasr, S. Anticoagulation use prior to common dental procedures: A systematic review. Cardiol. Res. Pract. 2019, 2019, 9308631. [CrossRef] [PubMed]

15. Tricco, A.C.; Lillie, E.; Zarin, W.; O’Brien, K.; Colquhoun, H.; Levac, D.; Moher, D.; Peters, M.D.J.; Horsley, T.; Weeks, L.; et al. PRISMA extension for scoping reviews (PRISMA-ScR): Checklist and explanation. Ann. Intern. Med. 2018, 169, 467-473. [CrossRef] [PubMed]

16. Munn, Z.; Stern, C.; Aromataris, E.; Lockwood, C.; Jordan, Z. What kind of systematic review should I conduct? A proposed typology and guidance for systematic reviewers in the medical and health sciences. BMC Med. Res. Methodol. $2018,18,5$. [CrossRef] [PubMed]

17. Leaper, D.J. Recent advances in surgery 26. I. Taylor and C. Johnson (eds). $155 \times 233$ mm. Pp. 241. Illustrated. 2003. Royal Society of Medicine Press: London. Br. J. Surg. 2004, 91, 375. [CrossRef]

18. Poli, P.P.; Beretta, M.; Cicciù, M.; Maiorana, C. Surgical and pharmacological treatments in the management of non-resorbable membrane and titanium mesh exposure following alveolar bone augmentation: A systematic scoping review. Open Dent. J. 2014, 8, 148-158. [CrossRef] [PubMed]

19. Al-Mubarak, S.; Al-Ali, N.; Abou-Rass, M.; Al-Sohail, A.; Robert, A.; Al-Zoman, K.; Al-Suwyed, A.; Ciancio, S. Evaluation of dental extractions, suturing and INR on postoperative bleeding of patients maintained on oral anticoagulant therapy. Br. Dent. J. 2007, 203, E15. [CrossRef] [PubMed]

20. Sacco, R.; Sacco, M.; Carpenedo, M.; Mannucci, P.M. Oral surgery in patients on oral anticoagulant therapy: A randomized comparison of different intensity targets. Oral Surg. Oral Med. Oral Pathol. Oral. Radiol Endod. 2007, 104, 18-21. [CrossRef]

21. Queiroz, S.I.M.L.; Silvestre, V.D.; Soares, R.M.; Campos, G.B.P.; Germano, A.R.; da Silva, J.S.P. Tranexamic acid as a local hemostasis method after dental extraction in patients on warfarin: A randomized controlled clinical study. Clin. Oral Investig. 2018, 22, 2281-2289. [CrossRef] [PubMed]

22. Mehm, P.; Cottrell, D.I.A.; Bestgen, S.C.; Booth, D.F. Management of Heparin Therapy in the High-Risk, Chronically Anticoagulated, Oral Surgery Patient: A Review and a Proposed Nomogram. J. Oral Maxillofac. Surg. 2000, 58, $198-202$.

23. Al-Mubarak, S.; Rass, M.A.; Alsuwyed, A.; Alabdulaaly, A.; Ciancio, S. Thromboembolic risk and bleeding in patients maintaining or stopping oral anticoagulant therapy during dental extraction. J. Thromb. Haemost. 2006, 4, 689-691. [CrossRef]

24. Al-Belasy, F.A.; Amer, M.Z. Hemostatic Effect of n-Butyl-2-Cyanoacrylate (Histoacryl) Glue in Warfarin-Treated Patients Undergoing Oral Surgery. J. Oral Maxillofac. Surg. 2003, 61, 1405-1409. [CrossRef] [PubMed] 
25. Da Silva, R.V.; Gadelha, T.B.; Luiz, R.R.; Torres, S.R. Intra-alveolar epsilon-aminocaproic acid for the control of post-extraction bleeding in anticoagulated patients: Randomized clinical trial. Int. J. Oral Maxillofac. Surg. 2018, 47, 1138-1144. [CrossRef] [PubMed]

26. Soares, E.C.S.; Costa, F.W.G.; Bezerra, T.P.; Nogueira, C.B.P.; DE Barros Silva, P.; Botelho Batista, S.H.; Sousa, F.B.; Fonteles, C.S. Postoperative hemostatic efficacy of gauze soaked in tranexamic acid, fibrin sponge, and dry gauze compression following dental extractions in anticoagulated patients with cardiovascular disease: A prospective, randomized study. Oral Maxillofac. Surg. 2015, 19, 209-216. [CrossRef] [PubMed]

27. Sammartino, G.; Marenzi, G.; Miro, A.; Ungaro, F.; Nappi, A.; Sammartino, P.; Josè, C.; Quaglia, F.; Mortellaro, C. Local delivery of the hemostatic agent tranexamic acid in chronically anticoagulated patients. J. Craniofac. Surg. 2012, 23, 648-652. [CrossRef]

28. Karsl, E.D.; Erdogan, Ö.; Esen, E.; Acartürk, E. Comparison of the effects of warfarin and heparin on bleeding caused by dental extraction: A clinical study. J. Oral Maxillofac. Surg. 2011, 69, 2500-2507. [CrossRef]

29. Bajkin, B.V.; Bajkin, I.A.; Petrovic, B.B. The effects of combined oral anticoagulant-aspirin therapy in patients undergoing tooth extractions: A prospective study. J. Am. Dent. Assoc. 2012, 143, 771-776. [CrossRef]

30. Bajkin, B.V.; Popovic, S.L.; Selakovic, S.D.J. Randomized, Prospective Trial Comparing Bridging Therapy Using Low-MolecularWeight Heparin With Maintenance of Oral Anticoagulation During Extraction of Teeth. J. Oral Maxillofac. Surg. 2009, 67, 990-995. [CrossRef]

31. Scarano, A.; Sinjari, B.; Murmura, G.; Mijiritsky, E.; Iaculli, F.; Mortellaro, C.; Tetè, S. Hemostasis control in dental extractions in patients receiving oral anticoagulant therapy: An approach with calcium sulfate. J. Craniofac. Surg. 2014, 25, 843-846. [CrossRef]

32. Bajkin, B.V.; Selaković, S.D.; Mirković, S.M.; Šarčev, I.N.; Tadić, A.J.; Milekić, B.R. Poređenje efikasnosti različitih metoda lokalne hemostaze kod pacijenata na oralnoj antikoagulantnoj terapiji posle ekstrakcije zuba. Vojnosanit. Pregl. 2014, 71, $1097-1101$. [CrossRef]

33. Halfpenny, W.; Fraser, J.S.; Adlam, D.M. Comparison of 2 hemostatic agents for the prevention of postextraction hemorrhage in patients on anticoagulants. Oral Surg. Oral Med. Oral Pathol. Oral Radiol. Endod. 2001, 92, 257-259. [CrossRef]

34. Evans, I.L.; Sayers, M.S.; Gibbons, A.J.; Price, G.; Snooks, H.; Sugar, A.W. Can warfarin be continued during dental extraction? Results of a randomized controlled trial. Br. J. Oral Maxillofac. Surg. 2002, 40, 248-252. [CrossRef] [PubMed]

35. Carter, G.; Goss, A.; Lloyd, J.; Tocchetti, R. Tranexamic Acid Mouthwash Versus Autologous Fibrin Glue in Patients Taking Warfarin Undergoing Dental Extractions: A Randomized Prospective Clinical Study. J. Oral Maxillofac. Surg. 2003, 61, 1432-1435. [CrossRef]

36. Carter, G.; Goss, A.N. Tranexamic acid mouthwash-A prospective randomized study of a 2-day regimen vs. 5-day regimen to prevent postoperative bleeding in anticoagulated patients requiring dental extractions. Int. J. Oral Maxillofac. Surg. 2003, 32, 504-507. [CrossRef] [PubMed]

37. Rocha, A.L.; Oliveira, S.R.; Souza, A.F.; Travassos, D.V.; Abreu, L.G.; Ribeiro, D.D.; Silva, T.A. Bleeding assessment in oral surgery: A cohort study comparing individuals on anticoagulant therapy and a non-anticoagulated group. J. Cranio Maxillofac. Surg. 2019, 47, 798-804. [CrossRef]

38. Mauprivez, C.; Khonsari, R.H.; Razouk, O.; Goudot, P.; Lesclous, P.; Descroix, V. Management of dental extraction in patients undergoing anticoagulant oral direct treatment: A pilot study. Oral Surg. Oral Med. Oral Pathol. Oral Radiol. 2016, 122, e146-e155. [CrossRef] [PubMed]

39. Bacci, C.; Maglione, M.; Favero, L.; Perini, A.; Di Lenarda, R.; Berengo, M.; Zanon, E. Management of dental extraction in patients undergoing anticoagulant treatment: Results from a large, multicentre, prospective, case-control study. Thromb. Haemost. 2010, 104, 972-975. [CrossRef] [PubMed]

40. Ockerman, A.; Vanhaverbeke, M.; Miclotte, I.; Belmans, A.; Vanassche, T. Tranexamic acid to reduce bleeding after dental extraction in patients treated with non-vitamin K oral anticoagulants: Design and rationale of the Extract-Noac trial. Br. J. Oral Maxillofac. Surg. 2019, 57, P1107-P1112. [CrossRef]

41. Cocero, N.; Basso, M.; Grosso, S.; Carossa, S.S.C. Direct Oral Anticoagulants and Medical Comorbidities in Patients Needing Dental Extractions: Management of the Risk of Bleeding. J. Oral Maxillofac. Surg. 2018, 77, P463-P470. [CrossRef]

42. Miller, S.G.; Miller, C.S. Direct oral anticoagulants: A retrospective study of bleeding, behavior, and documentation. Oral Dis. 2018, 77, 243-248. [CrossRef]

43. Lababidi, E.; Breik, O.; Savage, J.; Engelbrecht, H.; Kumar, R.; Assessing, C.W.C. Assessing an oral surgery specific protocol for patients on direct oral anticoagulants: A retrospective controlled cohort study. Int. J. Oral Maxillofac. Surg. 2018, 47, P940-P946. [CrossRef] [PubMed]

44. Patel, J.P.; Woolcombe, S.A.; Patel, R.K.; Obisesan, O.; Roberts, L.N.; Bryant, C.; Arya, R. Managing direct oral anticoagulants in patients undergoing dentoalveolar surgery. Br. Dent. J. 2017, 222, 245-249. [CrossRef]

45. Miclotte, I.; Vanhaverbeke, M.; Agbaje, J.O. Pragmatic approach to manage new oral anticoagulants in patients undergoing dental extractions: A prospective case-control study. Clin. Oral Investig. 2017, 21, 2183-2188. [CrossRef]

46. Hanken, H.; Gröbe, A.; Heiland, M.; Smeets, R.; Kluwe, L.; Wikner, J.; Koehnke, R.; Al-Dam, A.; Eichhprn, W. Postoperative bleeding risk for oral surgery under continued rivaroxaban anticoagulant therapy. Clin. Oral Investig. 2016, 20, 1279-1282. [CrossRef]

47. Rubino, R.T.; Dawson, D.R.; Kryscio, R.J.; Al-sabbagh, M.; Miller, C.S. Postoperative bleeding associated with antiplatelet and anticoagulant drugs: A retrospective study. Oral Surg. Oral Med. Oral Pathol. Oral Radiol. 2019, 128, 243-249. [CrossRef] 
48. Yoshikawa, H.; Yoshida, M.; Yasaka, M.; Yoshida, H.; Murasato, Y.; Fukunaga, D. Safety of tooth extraction in patients receiving direct oral anticoagulant treatment versus warfarin: A prospective observation study. Int. J. Oral Maxillofac. Surg. 2019, 48, 1102-1108. [CrossRef]

49. Caliskan, M.; Tükel, H.; Benlidayi, M.; Deniz, A. Is it necessary to alter anticoagulation therapy for tooth extraction in patients taking direct oral anticoagulants? Med. Oral Patol. Oral Cir. Bucal. 2017, 22, e767-e773. [CrossRef]

50. Neukam, F.W.; Rusche, B.; Bauersachs, A.; Musazada, S.; Schmitt, C.M. Management of anticoagulated patients in implant therapy: A clinical comparative study. Clin. Oral Imp. Res. 2015, 27, 1-9. [CrossRef]

51. Miranda, M.; Martinez, L.S.; Franco, R.; Forte, V.; Bollero, P. Differences between warfarin and new oral anticoagulants in dental clinical practice. Oral Implantol. 2016, 9, 151-156.

52. Yagyuu, T.; Kawakami, M.; Ueyama, Y.; Imada, M.; Kurihara, M.; Matsusue, Y.; Imai, Y.; Yamamoto, K.; Kirita, T. Risks of postextraction bleeding after receiving direct oral anticoagulants or warfarin: A retrospective cohort study. Dent. Oral Med. Res. 2017, 7, 1-7. [CrossRef] [PubMed]

53. Wahl, M.J.; Pinto, A.; Kilham, J.; Lalla, R.V. Dental surgery in anticoagulated patients—Stop the interruption. Oral Surg. Oral Med. Oral Pathol. Oral Radiol. 2015, 119, 136-157. [CrossRef]

54. Nikolakopoulos, I.; Spyropoulos, A.C. Heparin Bridging Therapy for Patients on Chronic Oral Anticoagulants in Periprocedural Settings. Semin. Thromb. Hemost. 2020, 46, 26-31. [CrossRef]

55. Fortier, K.; Shroff, D.; Reebye, U.N. Review: An overview and analysis of novel oral anticoagulants and their dental implications. Gerodontology 2018, 35, 78-86. [CrossRef]

56. Brennan, Y.; Gu, Y.; Schifter, M.; Crowther, H.; Favaloro, E.J.; Curnow, J. Dental extractions on direct oral anticoagulants vs. warfarin: The DENTST study. Res. Pract. Thromb. Haemost. 2020, 4, 278-284. [CrossRef] [PubMed]

57. Thomas, M.V.; Puleo, D.A.; Al-Sabbagh, M. Calcium sulfate: A review. J. Long Term Eff. Med. Implants. 2005, 15, 599-607. [CrossRef]

58. Pérez, M.; Fernández, I.; Márquez, D.; Guerra Bretaña, R.M. Use of N-butyl-2-cyanoacrylate in oral surgery: Biological and clinical evaluation. Artif. Organs 2000, 24, 241-243. [CrossRef] [PubMed]

59. Dunn, C.J.; Goa, K.L. Tranexamic Acid A Review of its Use in Surgery and Other Indications; Adis International Limited: Auckland, New Zealand, 1999. 